\title{
Accesibilidad territorial a centros ecoturísticos a partir de evaluación individual y centros de gravedad poblacional en Manizales, Colombia
}

\author{
Diego A. Escobar ${ }^{1}$, Jorge A. Montoya ${ }^{1}$ y Carlos A. Moncada ${ }^{2}$ \\ (1) Universidad Nacional de Colombia - Sede Manizales. Facultad de Ingeniería y Arquitectura, Departamento de \\ Ingeniería Civil, Grupo de Investigación en Movilidad Sostenible - GIMS. Campus La Nubia, Bloque S2-208, Carrera 37 \\ con Calle 94, Manizales, 170003, Colombia. (correo-e: daescobarga@unal.edu.co; joamontoyago@unal.edu.co) \\ (2) Universidad Nacional de Colombia - Sede Bogotá. Facultad de Ingeniería, Departamento de Ingeniería Civil y \\ Agrícola, Programa de Investigación en Tránsito y Transporte - PIT. Ciudad Universitaria edificio 214, oficina 417, \\ Bogotá, 111321, Colombia. (correo-e: camoncadaa@unal.edu.co)
}

Recibido Oct. 14, 2019; Aceptado Dic. 18, 2019; Versión final Feb. 10, 2020, Publicado Oct. 2020

\begin{abstract}
Resumen
El objetivo de esta investigación es establecer las condiciones de accesibilidad territorial desde y hacia elb conjunto de ecoparques existentes en la zona urbana de la ciudad de Manizales, a partir de análisis colectivos e individuales, mediante la aplicación de modelos geoestadísticos. La metodología emplea la accesibilidad territorial como elemento de evaluación, complementado con un análisis de cobertura poblacional de los centros de gravedad de cada comuna. Se vinculan variables de población y área como elementos de comparación de la accesibilidad territorial de la red de infraestructuras del transporte. Los resultados muestran que gran parte de la ciudad podría acceder o desplazarse a los ecoparques en un tiempo medio de viaje inferior a 25 minutos; no obstante, existen áreas con deficiencias en las condiciones de accesibilidad territorial. Se concluye que la metodología empleada permite identificar los sectores de la ciudad que refieren deficientes condiciones de accesibilidad territorial a este tipo de servicio, permitiendo direccionar proyectos que busquen salvaguardar estos espacios.
\end{abstract}

\section{Territorial accessibility to ecotourism centers from individual evaluation and population gravity centers in Manizales, Colombia}

\begin{abstract}
The objective of this research is to establish the conditions of territorial accessibility to and from all existing ecoparks in the urban area of the city of Manizales, based on collective and individual analyzes, by the application of geostatistical models. The methodology uses territorial accessibility as an assessment element. This is complemented by a coverage population analysis based on the centers of gravity of each commune. Population and area variables are linked as elements of comparison of territorial accessibility offered by the transport infrastructure network. The results show that a large part of the city can access or move to ecoparks in an average travel time of less than 25 minutes. However, there are areas with deficiencies in territorial accessibility conditions. It is concluded that the methodology used allows identifying the sectors of the city that report poor territorial accessibility conditions to this type of service, which enables to direct projects that seek to safeguard these spaces.
\end{abstract}

Keywords: ecopark; accessibility; gravity centers; population; coverage 


\section{INTRODUCCIÓN}

El proceso de expansión y fortalecimiento social y particular de un individuo, se encuentra fuertemente influenciado a la capacidad de interactuar y comprender el entorno circundante, propiciando elementos de alto impacto que permitan la construcción de ciudad (Franco y Zabala, 2012). A pesar de esto, los procesos de interacción actuales, han dejado de lado importantes aspectos relacionados con el uso de espacios ambientales y ambientales-recreativos, direccionándose más hacia comportamientos consumistas, que afectan el equilibrio social y ambiental, dificultando, cada vez más, la posibilidad de encontrar zonas libres de contaminación. Estos mencionados espacios ambientales y ambientales-recreativos, o más conocidos "Ecoparques", hacen referencia a aquellos espacios verdes, de libre interacción y focos de purificación de contaminantes al interior o exterior de la ciudad, en los cuales se estructuran, como pilar fundamental, la preservación de un medioambiente sano. La gran importancia de estos espacios ambientales-recreativos, conlleva a la necesidad de realizar estudios territoriales, con el fin de determinar posibles áreas de la ciudad que deban ser intervenidas para que se mejoren las condiciones de accesibilidad territorial (en términos de tiempo medio de viaje) y condiciones de cobertura poblacional. Es por esta razón, que en la presente investigación se plantea un análisis de accesibilidad territorial, mediante transporte automotor, hacia el conjunto de ecoparques existentes en la ciudad de Manizales, con el fin de establecer un precedente relacionado al análisis de zonas protegidas, debido a que, actualmente no se cuenta con un modelo evaluativo que vincule dicho concepto. La presente investigación, evalúa la relación existente entre la red de infraestructuras del transporte y la localización de los ecoparques, a través de un enfoque geográfico (Cao, et al., 2015); no obstante, algunos modelos actuales de evaluación se desarrollan como medidas de aplicación de políticas de conservación en variados contextos (Thomasina, et al., 2004), las cuales vinculan diferentes disciplinas como el urbanismo (Van Lindert, 2016; Wu et al., 2019), la economía (Guzmán et al., 2020), la ingeniería (Newland et al., 2018), seguridad vial (Londoño et al., 2017), el turismo (Lau y McKerher, 2006; Paulino et al., 2019), entre otras.

Manizales, ciudad capital del departamento de Caldas, perteneciente a la denominada región andina colombiana, se ubica a $75^{\circ} 29^{\prime} 05^{\prime \prime}$ de longitud Oeste y a $5^{\circ} 3^{\prime} 58^{\prime \prime}$ de latitud Norte (Ver Figura 1). Su condición de alta montaña, situada sobre los 2150 m.s.n.m., le permite preservar zonas boscosas húmedas, con gran variedad de fauna y flora. La ciudad cuenta con una extensión total de $572 \mathrm{~km}^{2}$ (Escobar et al., 2018); de los cuales, solo el $6,14 \%\left(35,11 \mathrm{~km}^{2}\right)$ corresponden al área urbana donde habitan cerca de 372.578 habitantes según datos estadísticos de nivel nacional; adicionalmente, debido a su cercanía con el municipio de Villamaría, se estructura como una unidad conurbada con alta influencia económica, social y cultural (Escobar et al., 2018a), por lo tanto se hace sumamente importante incluir la superficie y red vial urbana de este municipio dentro del estudio. Villamaría, se encuentra ubicado al costado sur de Manizales (Ver Figura 1), sobre los $75^{\circ} 30^{\prime} 55^{\prime \prime}$ de longitud Oeste y $5^{\circ} 02^{\prime} 44^{\prime \prime}$ de latitud Norte, posee una extensión total de $462 \mathrm{~km}^{2}$ según datos oficiales de la administración municipal, y una población urbana estimada de 49.731 habitantes según datos estadísticos de nivel nacional. El comportamiento conurbado entre ambas municipalidades, es permitido por una única vía de conexión con cerca de $4 \mathrm{~km}$ de longitud, la cual se encuentra vinculada a la red de infraestructuras del transporte $(749 \mathrm{Km})$ del conjunto Manizales-Villamaría, la cual ha sido actualizada en base a la red utilizada en anteriores investigaciones (Escobar et al., 2018a; 2018b).

Una característica importante en el desarrollo humano, radica en la posibilidad del individuo de interactuar con el medio circundante, por lo tanto, al entender la conexión entre los municipios, es posible examinar la interacción de ambas poblaciones con los diferentes equipamientos existentes, dentro de los cuales, los ecoparques juegan un papel importante como herramienta para la formación social, cultural y ambiental de la comunidad en general. Son entonces los Ecoparques aquellos espacios radiantes y llenos de vida, entre horizontes de cemento, necesarios para la formación del individuo y disfrutados como bienes inmateriales de la población (Paquot, 2012), además de considerarse como una estrategia importante en la mejora de la calidad de vida de nuestras sociedades cada vez más urbanizadas y expandidas periféricamente (Unal, et al., 2016). Para el caso de Manizales, la definición de Ecoparques se encuentra abordada dentro de las áreas de interés ambiental urbanas, establecidas en el Plan de Ordenamiento Territorial (2017-2031) como aquellas zonas de importancia estratégica y de soporte que permiten la preservación, restauración o uso sostenible de la biodiversidad existente, así como los servicios ecosistémicos, limitadas a condiciones de uso y manejo, promoviendo la potencialización de valores desde una perspectiva pública y/o privada. Actualmente, la ciudad cuenta con 6 zonas protegidas a nivel urbano, las cuales varían en cuanto a extensión (Ver Figura 2) y características particulares (servicios, áreas funcionales, áreas deportivas, etc.), las cuales permiten la interacción de los habitantes con el medio ambiente; las áreas objeto de investigación son: Ecoparque Bosque Popular el Prado, Ecoparque Los Yarumos, Ecoparque Los Alcázares, Ecoparque Bosque Central Universitario, Ecoparque Cerro Sancancio y Ecoparque Bosque Monteleón. 


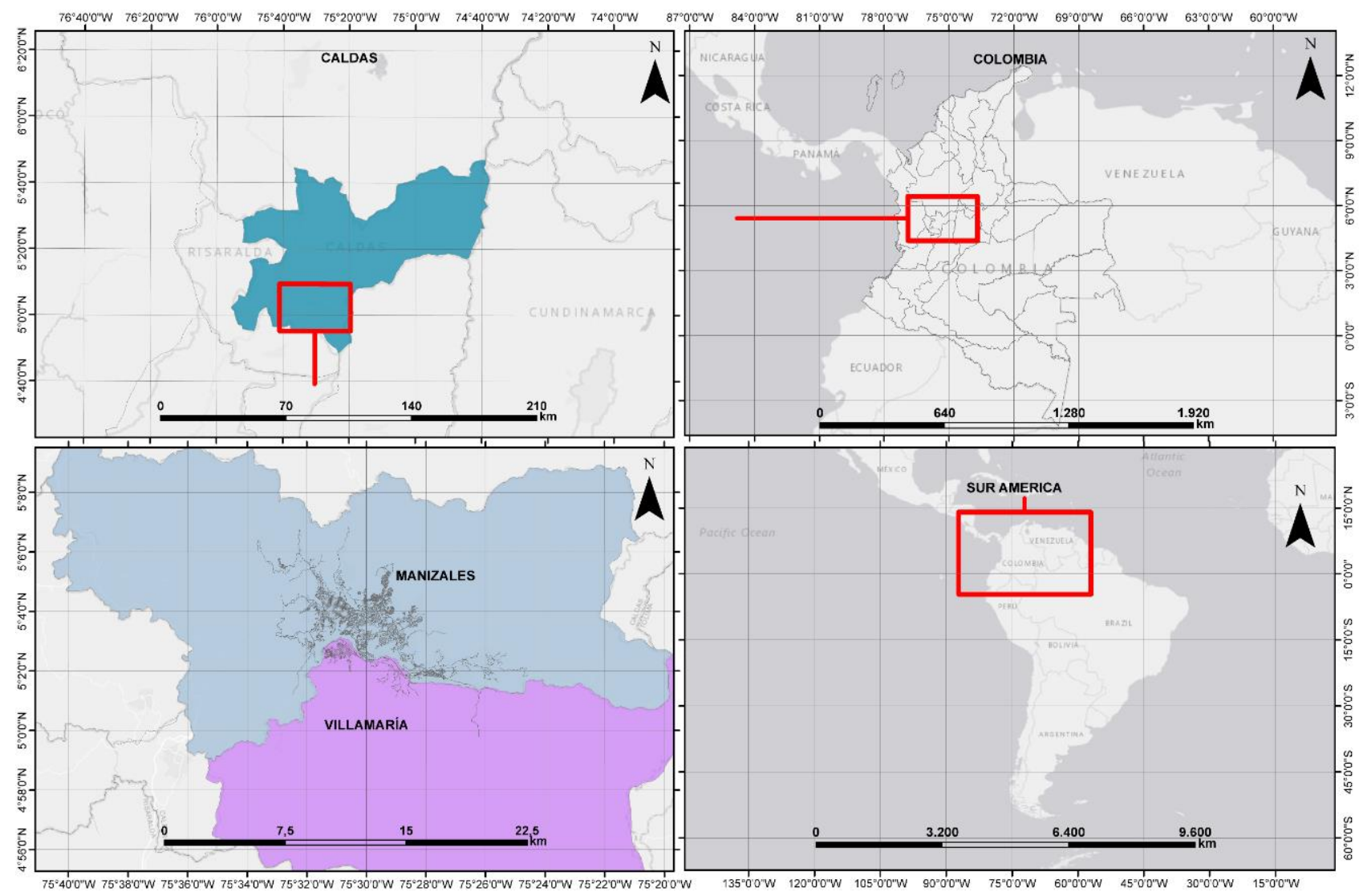

Fig. 1: Localización del área de estudio.

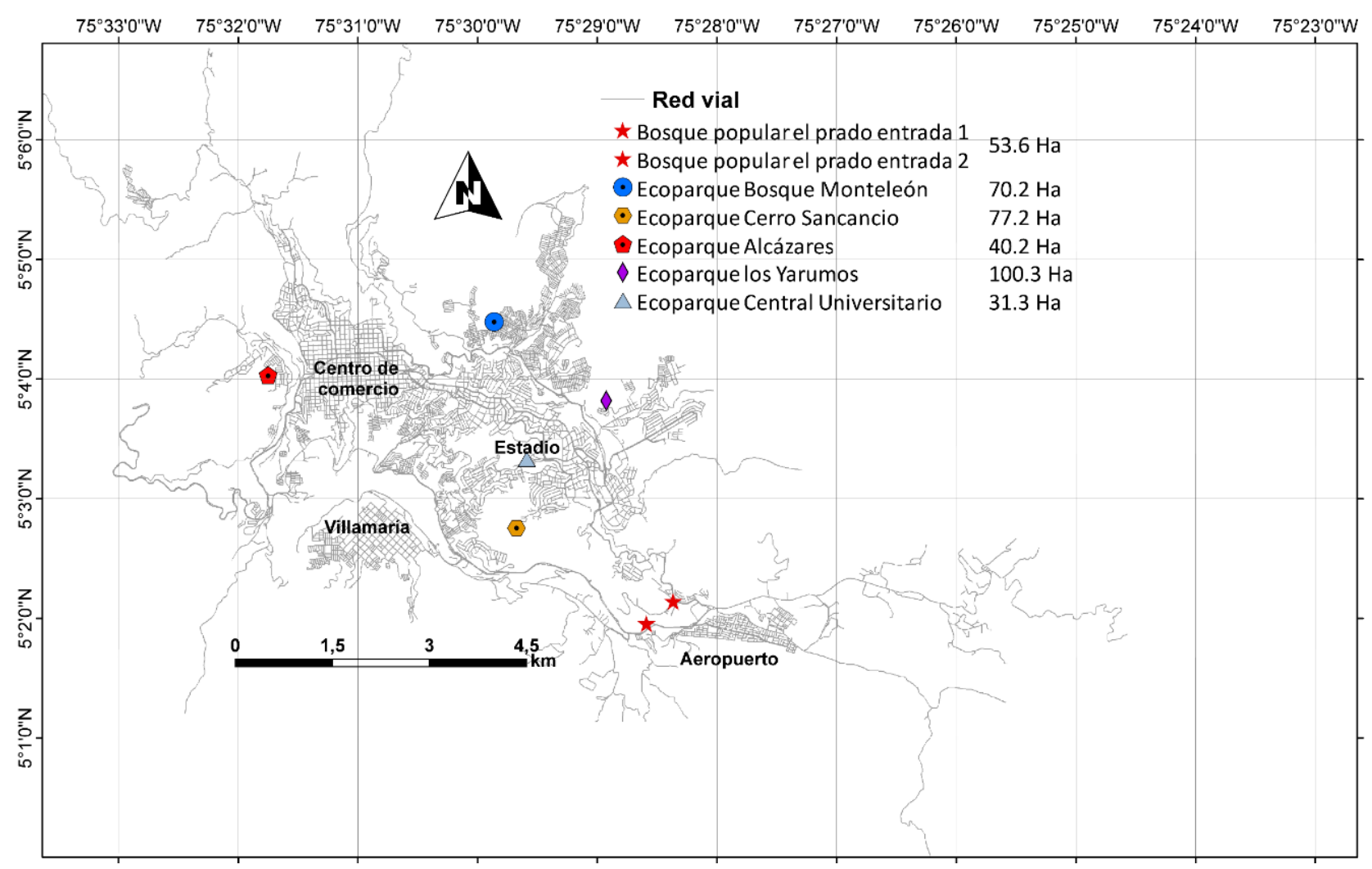

Fig. 2: Localización de ecoparques.

Como requerimiento previo al abordaje del estudio, se hace necesario incluir e interiorizar el termino accesibilidad territorial, el cual se define como "la relación entre formas básicas de actividad humana: movilidad, comprensión y comunicación; sujetas a limitaciones de alcanzarlas dadas la existencia de barreras" (Moncada et al., 2018); esta definición parte desde el concepto fundamental establecido por Hansen (1959) como "The potencial of oportunities for interaction", el cual ha sido base para investigaciones posteriores 
(Geurs y Van Wee, 2004; Vega, 2011); no obstante, a pesar de que, la definición base proviene del año 1959, se han tenido registros del uso del concepto desde principios del siglo XX (Batty, 2009). Dentro de esta formulación del término, se puede asumir entonces que, la accesibilidad territorial no es más que la medida de la facilidad con la cual un usuario se desplaza desde un nodo particular hacia algún destino específico sobre el cual debe realizar una actividad, teniendo en cuenta que, este desplazamiento es directamente afectado por el modo de transporte empleado. Algunos componentes o variables inmersas en la caracterización de la accesibilidad territorial, son: orígenes y destinos (Mozón de Cáceres, 1988), modos de transporte, nodos de actividad, velocidades de operación, distancia, entre otros; componentes con los cuales se han desarrollado numerosos estudios y aplicaciones en variados temas de investigación, tales como: Sostenibilidad (Bertolini, et al., 2005), usos del suelo y calidad del transporte (Boisjoly y Ahmed, 2017), planeación del transporte (Morris, et al., 1979; Mozón de Cáceres, 1988), exclusión social (Schürmann et al., 1997); etc.

\section{METODOLOGÍA}

La metodología de investigación empleada, se estructura en un total de cinco fases consecutivas, las cuales se presentan en la Figura 3 y se describen a continuación.

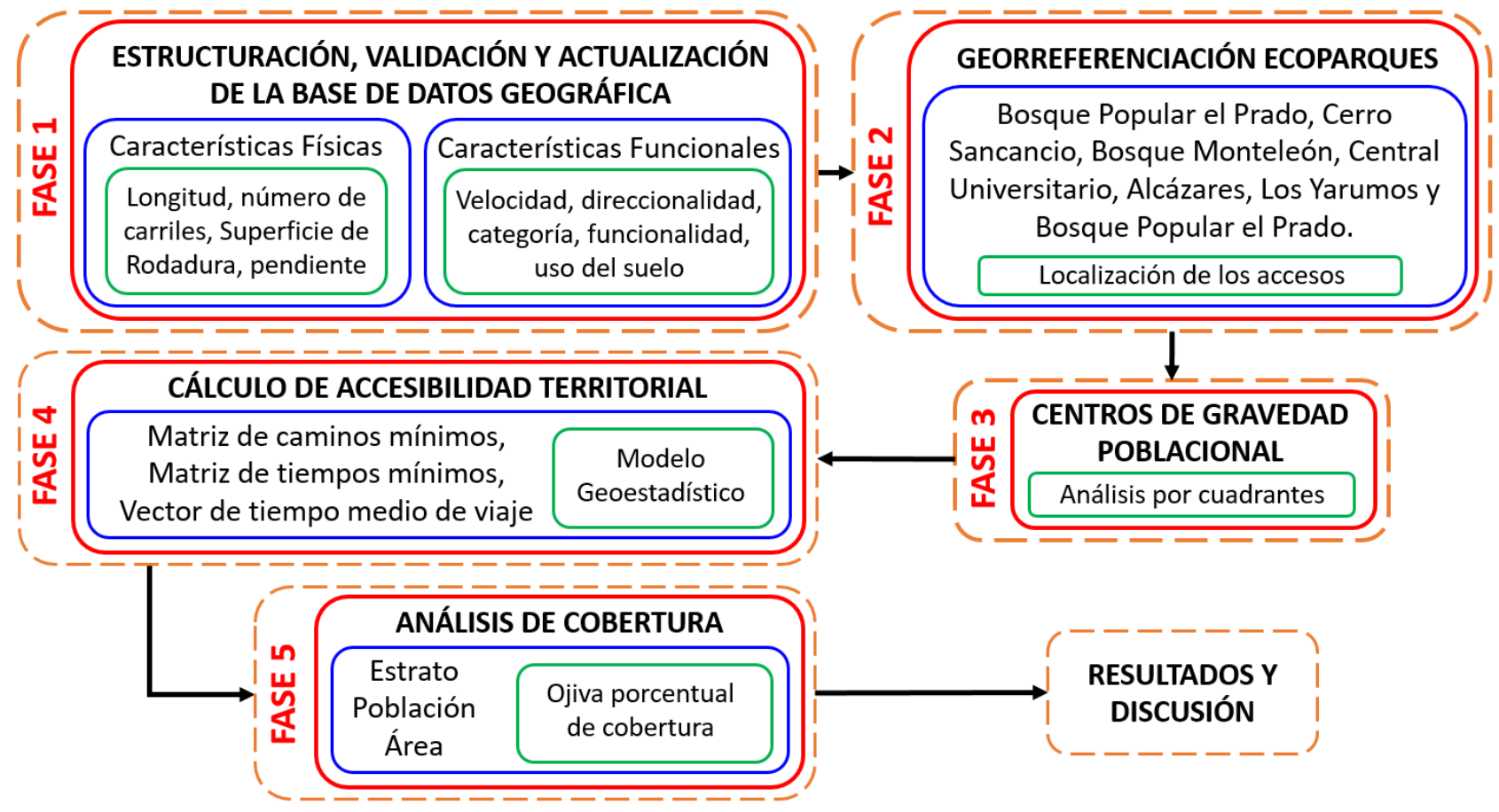

Fig. 3: Metodología de investigación.

\section{Fase 1. Estructuración, validación y actualización de la base de datos geográfica}

En primera instancia, se estructura y optimiza la red de infraestructura del transporte asociada al conjunto Manizales-Villamaría, la cual ha sido actualizada en base a la red utilizada en anteriores investigaciones (Escobar et al., 2018a; 2018b). La red está compuesta por un total de 11374 links y 8580 nodos, distribuidos espacialmente, además de incluir las características físicas y funcionales del sistema (direccionalidad, velocidad, longitud, clase, tipo de rodadura, etc); es importante aclarar que, la velocidad empleada contempla el promedio de las velocidades de los modos de transporte que circulan sobre la red vial, para nuestro caso particular, se analizaron los vehículos automotores (automóvil, motocicleta, transporte público colectivo urbano y transporte público individual). El funcionamiento de la red dentro de los cálculos, se encuentra sujeto al grado de detalle que presente, por lo tanto, mediante indagación de archivos se definen las diferentes intervenciones realizadas por la administración municipal en el periodo 2010-2019, dentro de los cuales están ampliaciones de vía, nuevos puentes, mejoramiento de la superficie de rodadura, entre otros. El proceso de estructuración y validación de la red, por ejemplo incluir en el grafo nuevas vías y verificar su existencia en campo, se realiza a partir de la herramienta digital ArcMap de Esri la cual permite realizar intervenciones a partir de la teoría de grafos, en la que se consideran las conexiones y extensiones de vía mediante nodos (puntos fijos que parametrizan los cruces viales) y arcos (conjunto de líneas que representan segmentos de vía asociados a la infraestructura vial) (Gross y Tucker, 1987). El conjunto de intervenciones realizadas, considera análisis de conectividad de la red, mediante la herramienta topology, así como correcciones pertinentes a partir de la barra de ediciones del software. 


\section{Fase 2. Georreferenciación de los ecoparques}

En esta fase se georreferencian los puntos de estudio (Ecoparques), a través de la herramienta ArcMap; en esta se localiza el punto de acceso a cada uno de estos. Esta fase es de vital importancia en los cálculos de accesibilidad territorial, considerando que, un usuario del entorno accede al centro ecoturístico al alcanzar el acceso permitido por la entidad encargada de la gestión del predio. Los puntos de interés ecoturístico de la zona de estudio son en total 6 (Ver Figura 2): Ecoparque Bosque Popular el Prado, Ecoparque Cerro Sancancio, Ecoparque Bosque Monteleón, Ecoparque Central Universitario, Ecoparque Alcázares, Ecoparque los Yarumos y Ecoparque Bosque Popular el Prado. Todos los ecoparques poseen un solo acceso dada la localización de la población circundante a los mismos, excepto el Ecoparque Bosque Popular el Prado, el cual cuenta con 2 accesos que permiten el ingreso de los usuarios procedentes el sector norte-occidente de la ciudad, además de un acceso en el entorno sur, como acceso de la población residente en el entorno oriental y sur.

\section{Fase 3. Centros de gravedad poblacional}

Esta etapa consiste en ponderar la población existente en cada manzana habitada de la ciudad por su respectiva longitud y latitud, Ecuación (1) y Ecuación (2), con lo cual, al ser dividido sobre el total poblacional de la comuna correspondiente, se obtiene el centro de gravedad de la misma (Ver Tabla 2).

$$
\begin{aligned}
& \text { Longitud }_{i}=\frac{\sum_{j=1}^{n} \text { Población }_{j} * \text { Longitud }_{j}}{\sum \text { Población }} \\
& \text { Latitud }_{i}=\frac{\sum_{j=1}^{n} \text { Población }_{j} * \text { Latitud }_{j}}{\sum \text { Pob lación }}
\end{aligned}
$$

Tabla 1. Localización de los centros de gravedad poblacional de cada comuna

\begin{tabular}{|c|c|c|c|c|}
\hline FID & longitud & latitud & comuna & Tipo \\
\hline 18 & 1171901,65 & 1053313,01 & 1 & Densidad Poblacional \\
\hline 20 & 1173434,26 & 1052992,84 & 2 & Densidad Poblacional \\
\hline 0 & 1173108,29 & 1052313,95 & 3 & Densidad Poblacional \\
\hline 2 & 1175110,85 & 1052110,91 & 4 & Densidad Poblacional \\
\hline 4 & 1175926,06 & 1053280,68 & 5 & Densidad Poblacional \\
\hline 7 & 1177535,89 & 1051372,00 & 6 & Densidad Poblacional \\
\hline 9 & 1179081,26 & 1048498,54 & 7 & Densidad Poblacional \\
\hline 10 & 1176312,63 & 1050852,73 & 8 & Densidad Poblacional \\
\hline 12 & 1174855,70 & 1050365,14 & 9 & Densidad Poblacional \\
\hline 14 & 1173982,96 & 1051500,14 & 10 & Densidad Poblacional \\
\hline 16 & 1172468,10 & 1051570,79 & 11 & Densidad Poblacional \\
\hline 23 & 1173389,97 & 1049673,64 & 99 & Densidad Poblacional \\
\hline
\end{tabular}

Posteriormente, se definen el conjunto de líneas perpendiculares con origen en el centro de gravedad que conforman los cuadrantes definiéndose así los cuadrantes de cada una de las 11 comunas de la ciudad (Ver Figura 4), adicionando el conjunto de Villamaría; por otra parte, en la figura 6, se aprecia la localización de las once comunas que conforman la ciudad de Manizales, así como la localización del municipio contiguo de Villamaría.

\section{Fase 4. Cálculo de accesibilidad territorial}

En la tercera etapa metodológica se definen los elementos fundamentales para la elaboración de las curvas de accesibilidad territorial, cuyo cálculo se realiza a partir del vector de tiempo mínimo de viaje, en donde se establece la impedancia de transitar sobre un arco i mediante la ecuación (3), donde Tvi es el tiempo de viaje del arco i, Li la longitud y Vi la velocidad del arco obtenida a partir de los modos de transporte en circulación. 

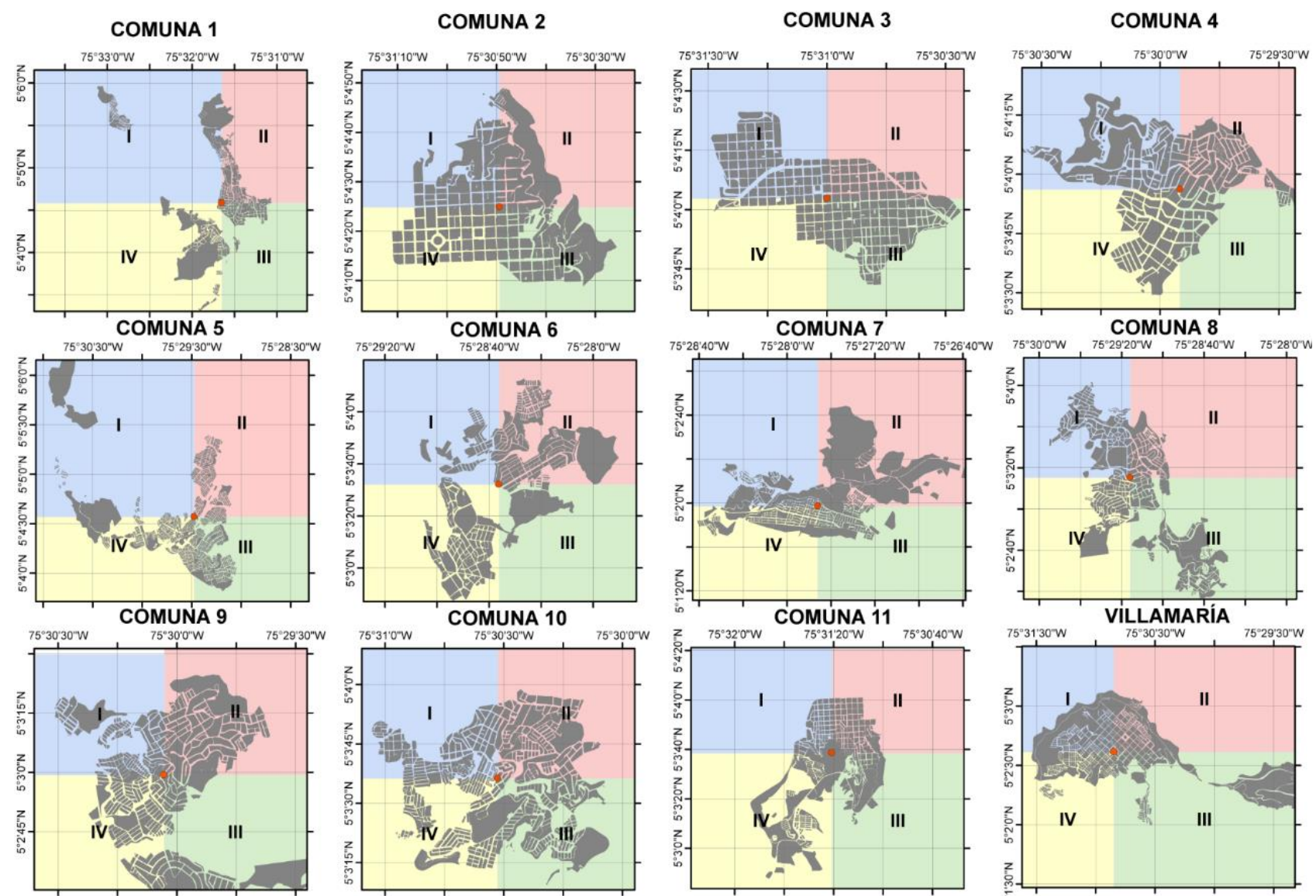

Fig. 4: Centros de gravedad y cuadrantes por comuna.

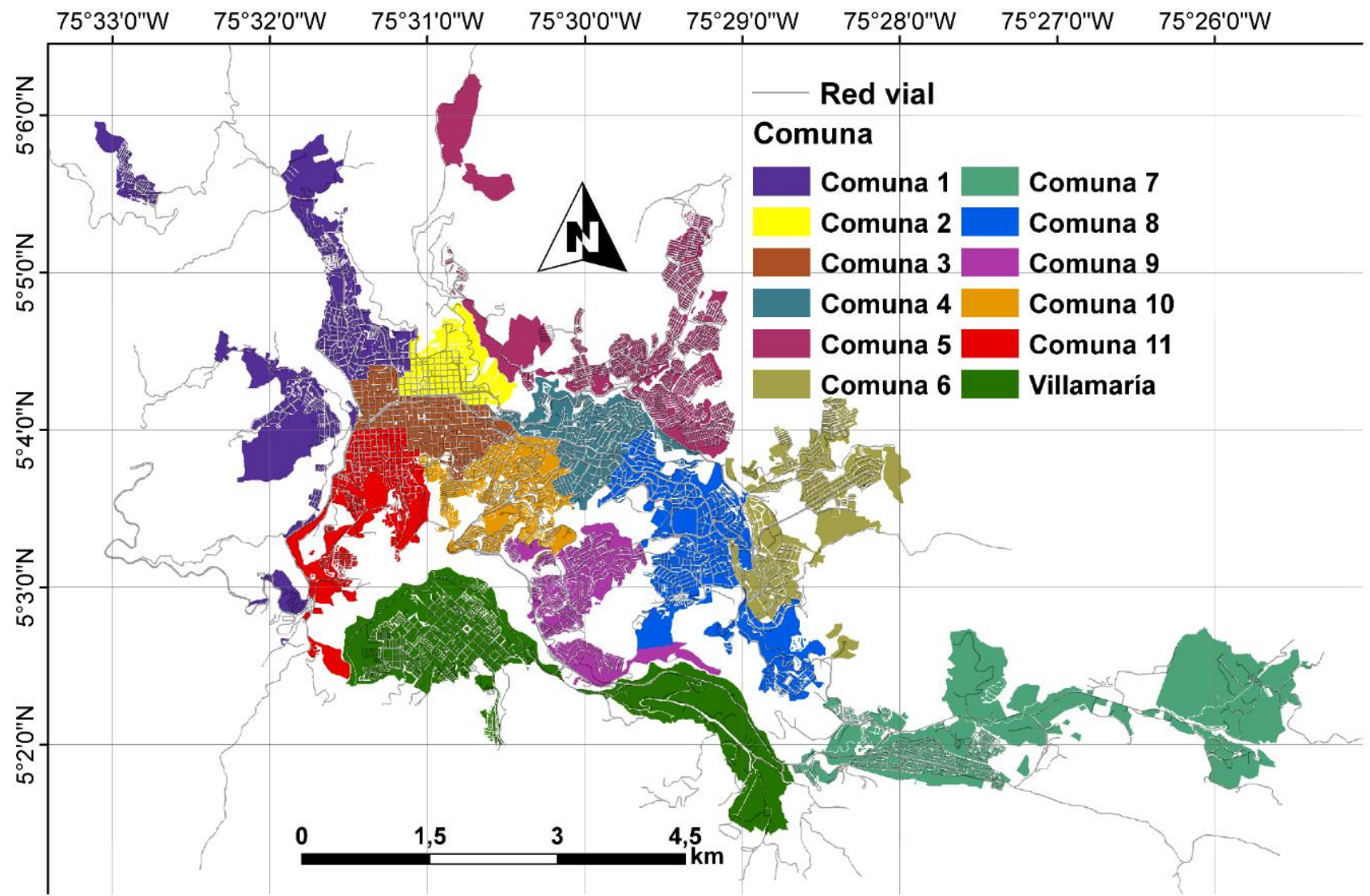

Fig. 5: Localización de las comunas.

$T v_{i}=\frac{L_{i}}{V_{i}} \quad i=1,2,3, \ldots, n$ 
El proceso de cálculo del vector de tiempo de viaje se fundamenta a partir del uso del algoritmo Dijkstra o más conocido como algoritmo de caminos mínimos, el cual determina el recorrido de menor impedancia en tiempo de viaje desde un nodo de origen $\mathrm{O}$ hasta un nodo de destino $\mathrm{F}$, transitando sobre nodos intermedios; a partir de lógica matemática de existencia de conexión (Dijstra, 1959). Posteriormente, se relacionan los datos geoespaciales de cada nodo para obtener así la matriz de tiempos mínimos de viaje, la cual se utiliza en la construcción de las curvas isócronas.

La construcción de las curvas se realiza mediante la herramienta ArcMap de Esri, a partir de la extensión Geostatical Wizard, esta extensión emplea el modelo de interpolación espacial Kriging ordinario con semivariograma lineal, con el cual se determinan las propiedades de dependencia espacial entre nodos de un mismo universo (ecuación 4), donde $\gamma_{(h)}$ es la semivarianza media en función de $\mathrm{h}, \mathrm{Z}(\mathrm{x})$ es el valor de la variable en el punto de coordenadas $x, y ; Z(x+h)$ el siguiente valor de la muestra a una distancia $h ; n$ es el número de elementos con una distancia $h$.

$$
\overline{\gamma_{(h)}}=\frac{\sum\left(Z_{(x+h)}-Z_{(x)}\right)^{2}}{2 n}
$$

Una vez obtenidas las curvas isócronas, es posible definir los sectores de la ciudad con deficiencias de acceso en los diferentes modos de transporte disponibles hacia el sistema de ecoparques; adicionalmente, y con el fin de complementar el ítem metodológico, se realiza la intervención asociada a los cuadrantes poblacionales, en donde, mediante la herramienta "Geoprocessing Intersect" de ArcMap, se superponen las curvas isócronas y los polígonos de cuadrantes de cada comuna; permitiendo discriminar el comportamiento de la accesibilidad territorial por cuadrantes relacionada a los Ecoparques.

\section{Fase 5. Análisis de cobertura}

Esta fase contempla la incorporación de las variables socioeconómicas al estudio, siendo estas, área, comunas, población y estratificación socioeconómica (Ver figura 6). Esta última variable se encuentra definida por el Departamento Administrativo Nacional de Estadista como una aproximación a la diferencia socioeconómica jerarquizada, dando a interpretar entonces como la brecha económica existente entre poblaciones de una misma localidad, en nuestro caso particular se cuenta con un total de 6 estratos socioeconómicos, en donde el estrato 1 es de menor capacidad económica, mientras que el estrato 6 es el de mayor capacidad económica, definidos éstos mediante análisis no sólo del estado y materiales de la vivienda sino también de las posibilidades económicas de los habitantes en ella.

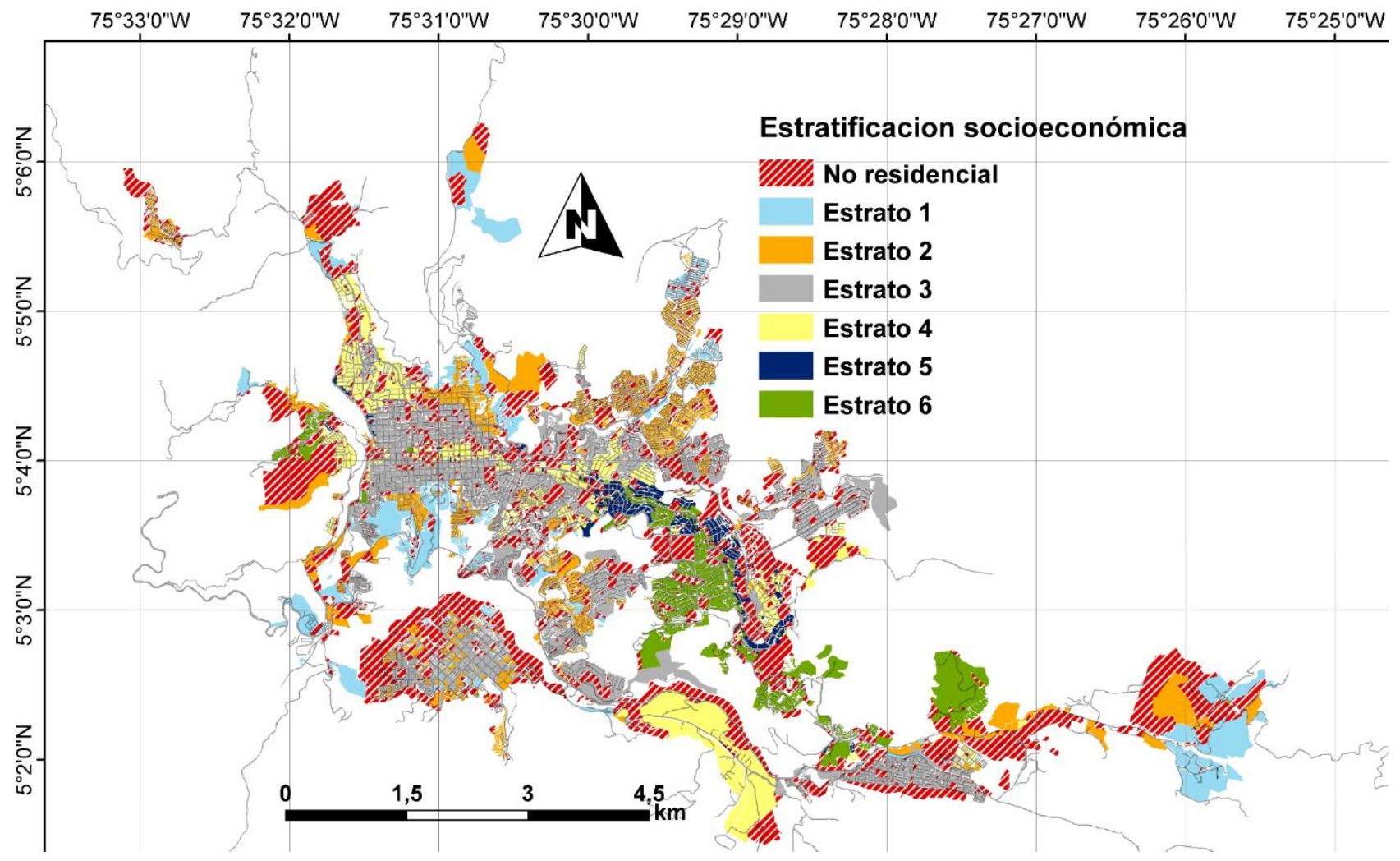

Fig. 6: Estratificación socioeconómica. 
De igual forma se vinculan en total 11 comunas, pertenecientes a la ciudad de Manizales, además de incluir el Municipio de Villamaría, como una comuna adicional (Ver figura 5); esto sumado a una distribución poblacional en la que altas densidades se presentan en la zona centro y bajas densidades en la zona occidente; y una distribución geográfica de la red de infraestructuras del transporte (Ver figura 2), serán de ayuda para una correcta interpretación de los datos.

El proceso de correlación de variables, emplea un modelo lineal simple con el fin de observar el grado de interacción entre el tiempo medio de viaje y el número de residentes cubiertos; por otra parte, el análisis de cobertura, se realiza mediante la superposición de capas con la información pertinente; en este caso se sobreponen los polígonos asociados a las curvas isócronas, estratificación socioeconómica y cuadrantes mediante la herramienta "intersect" perteneciente a la extensión Geoprocessing de Arcmap; posteriormente, la información calculada, se traslada a Microsoft Excel, en la cual se desarrolla el procedimiento de cálculo para obtener las curvas de cobertura porcentual. El manejo estadístico en la herramienta Excel, contempla la asociación de las variables cobertura y tiempo medio de viaje; por ejemplo, determinar cuánto porcentaje de población se cubre desde o hacia un Ecoparque si el tiempo medio de viaje es de 20 minutos.

\section{RESULTADOS Y DISCUSIÓN}

Luego de establecerse las condiciones necesarias relacionadas con la red de infraestructuras del transporte y variables socioeconómicas para el análisis de accesibilidad territorial, se estructuran los diferentes modelos de evaluación.

\section{Accesibilidad territorial desde y hacia el sistema de ecoparques}

Como resultado de la evaluación de la accesibilidad territorial desde y hacia el conjunto de ecoparques, se construye la figura 7 y la figura 8, en la cual se aprecia las curvas de tiempo medio de viaje (Tv) desde y hacia los 6 ecoparques, respectivamente, en intervalos de 5 minutos. Se observa, en gran medida, que el valor de acceso para la ciudad se encuentra en un tiempo medio de viaje inferior a los 25 minutos para ambos análisis; no obstante, existen sectores con valores de hasta 50 minutos. De igual forma, es posible afirmar, a partir de los tiempos de desplazamiento, que el sector oriente de la zona de estudio registra las mejores condiciones de accesibilidad territorial.

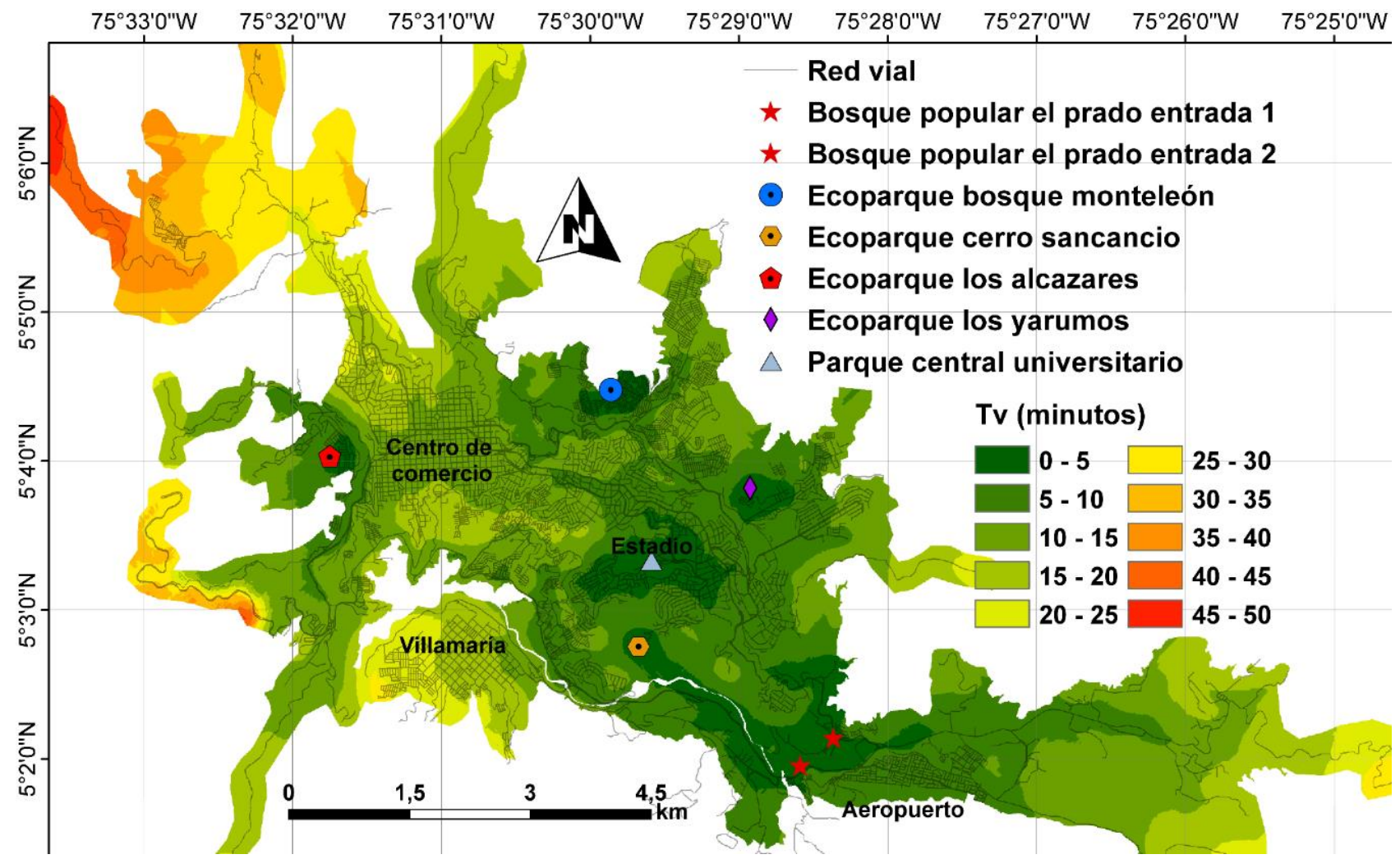

Fig. 7: Curvas de accesibilidad territorial desde el sistema de ecoparques. 


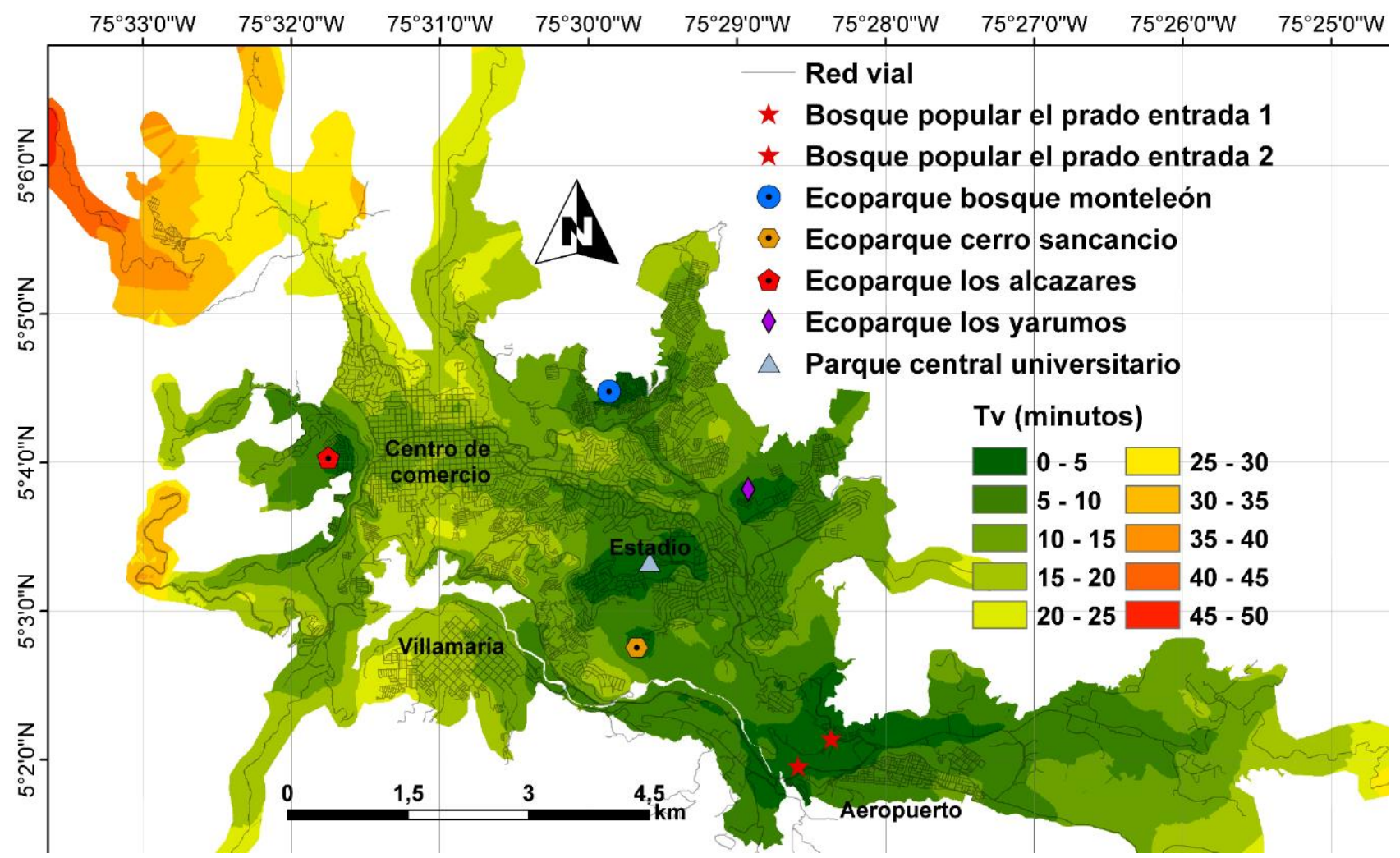

Fig. 8: Curvas de accesibilidad territorial hacia el sistema de ecoparques.

Por parte del análisis de cobertura poblacional y de área acumulada, se obtiene la figura 9; a partir de las curvas de accesibilidad territorial del conjunto de ecoparques. En ésta se observa un comportamiento raíz similar, inferior a 10 minutos, en el cual la variación de cobertura entre población y área no es significativa; posteriormente, en la parte a), se observa una cobertura cercana al $80 \%$ de la población en un tiempo de viaje inferior a 15 minutos, mientras que, la cobertura de área se encuentra en un valor cercano al $62 \%$ para el mismo límite de tiempo. De otro lado, en la parte b) de la figura 9, definiendo el mismo límite de tiempo (15 minutos), se logra cubrir alrededor del $70 \%$ de la población y $58 \%$ del área. A partir de estos resultados, se concluye que se posee mayor facilidad de desplazamiento desde los ecoparques, no obstante, la variación asociada en ambos escenarios presenta un comportamiento similar; de igual forma, es posible afirmar que el costo en tiempo de desplazamiento es favorable, si se compara con los requerimientos de tiempo en otras ciudades de Colombia y el mundo (Bogotá Como Vamos, 2017; MOOVIT, 2016), en las cuales suele superarse los 50 minutos.
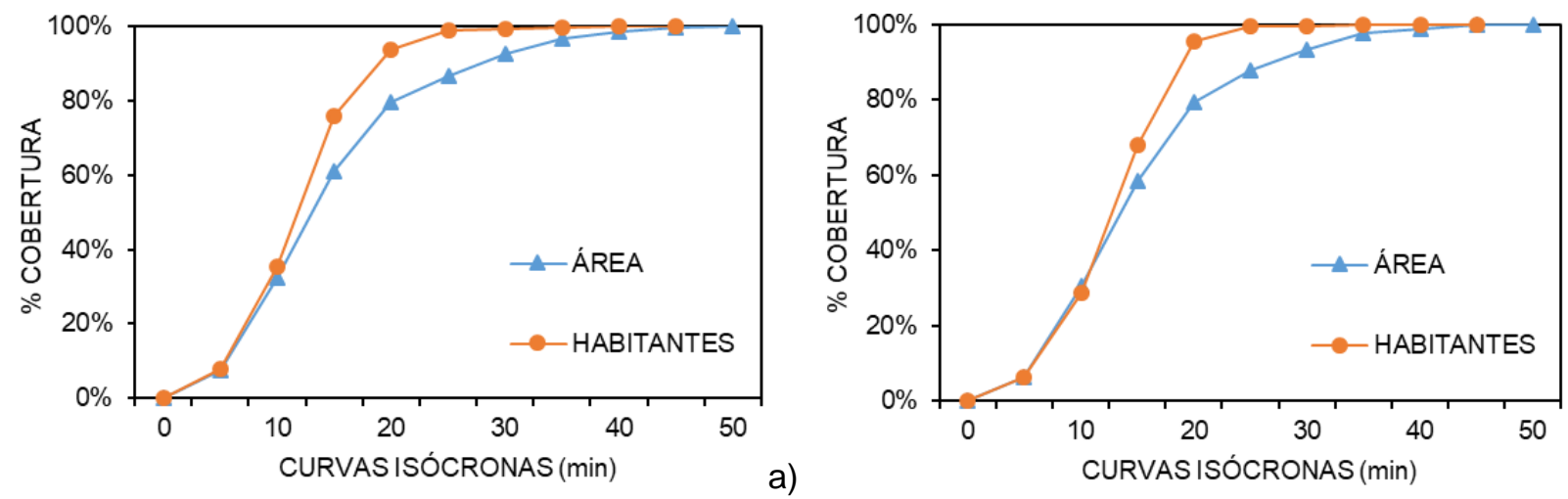

b)

Fig. 9: Porcentaje acumulado de cobertura poblacional y de área del sistema de ecoparques. a) desde los ecoparques; b) hacia los ecoparques.

Adicionalmente, en la figura 10, se presenta la variación en cobertura por estrato socioeconómico para los análisis desde y hacia los ecoparques; en la parte a), se identifica que, con un tiempo de viaje inferior a los 10 minutos desde los ecoparques, los estratos 5 y 6 suplen alrededor del $90 \%$ de su población, mientras los estratos 1, 2, 3 y 4 requieren de por lo menos 20 minutos. En la parte b) de la figura 10, se tiene un comportamiento similar para los estratos 5 y 6 al desplazarse hacia los ecoparques, no obstante, se presenta 
una reducción cercana al 10\% para el estrato 5; para los estratos restantes, se observa una conservación en su cobertura a excepción del estrato 1 el cual disminuye su cobertura al 50\% para un límite de tiempo de 15 minutos.
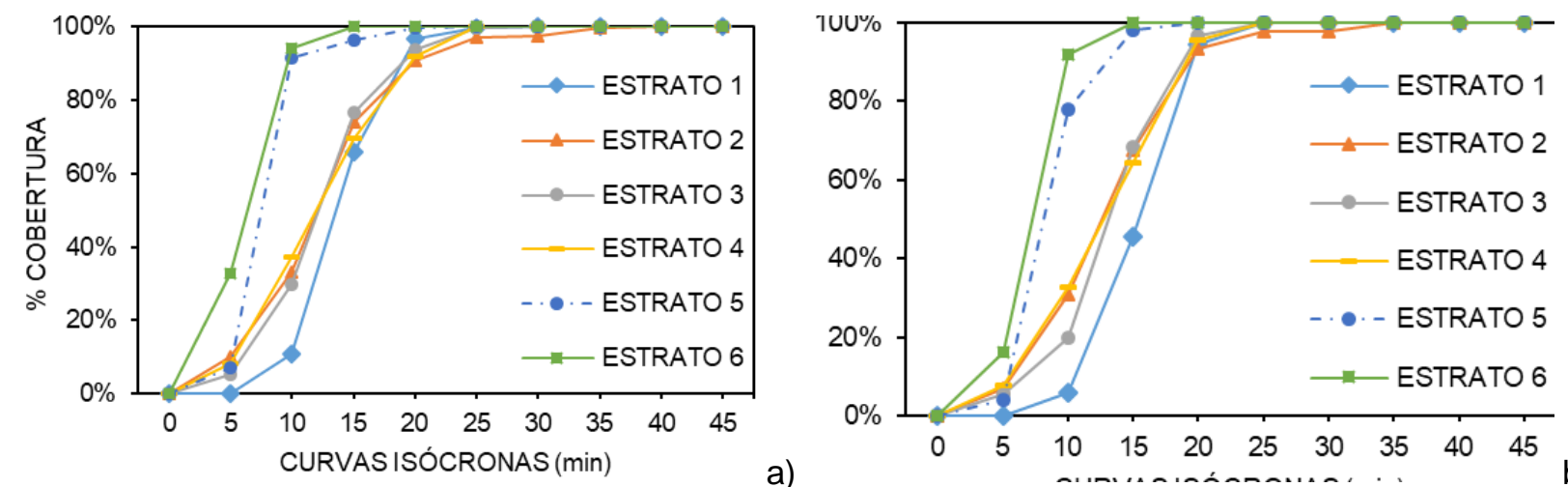

a)

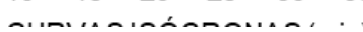

b)

Fig. 10: Porcentaje acumulado de cobertura por estrato socioeconómico. a) desde los ecoparques; b) hacia los ecoparques.

Ahora bien, realizando un análisis de correlación entre las variables tiempo medio de viaje, población y área, pero para el caso "desde el conjunto de ecoparques", en la figura 12 se observan los coeficientes obtenidos.
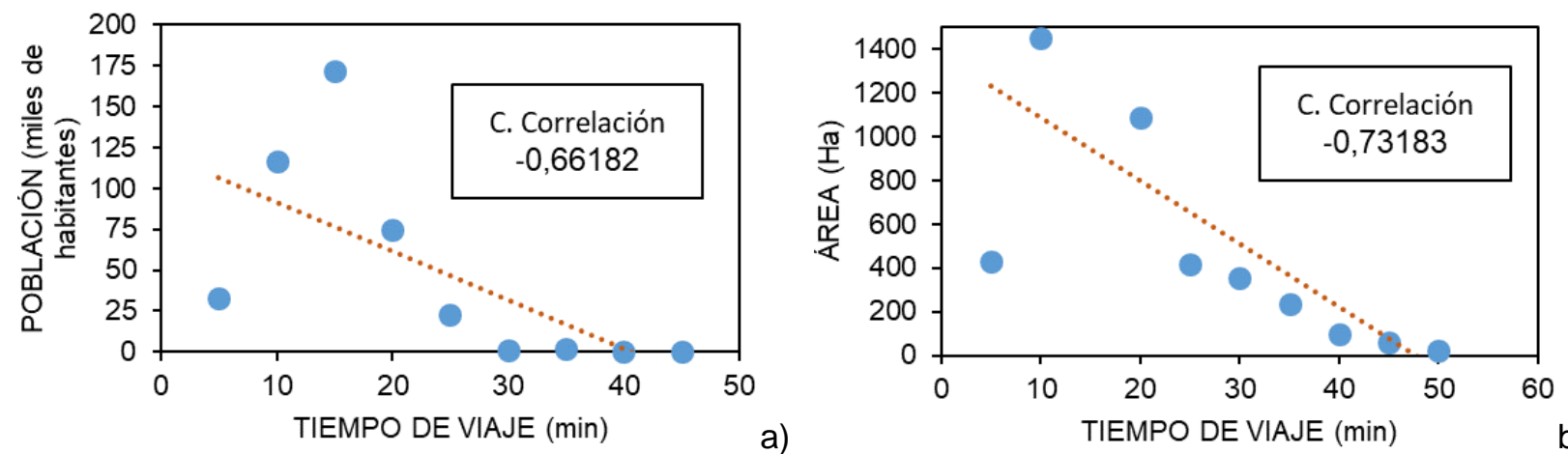

Fig. 11: Coeficientes de correlación, caso "desde los ecoparques", a) población vs tiempo medio de viaje; b) área vs tiempo medio de viaje.

El coeficiente de correlación entre las variables población y tiempo medio de viaje (Ver figura 11a), es de 0,66182, que representa una relación decreciente entre ambas variables, mientras el tiempo medio de viaje aumente, la población cubierta disminuye; igual comportamiento se observa al correlacionar las variables área y tiempo medio de viaje (Ver figura 11b), con un coeficiente de correlación de -0,73183. Para ambos casos se tiene que el coeficiente de correlación es considerado moderado, indicando que la relación entre tiempo medio de viaje y cobertura de las variables población y área dada la localización geoespacial de los ecoparques a pesar de ser decreciente, si existe y es significativa.

Por otra parte, realizando un análisis de correlación entre las variables tiempo medio de viaje, población y área, en la figura 12 se observan los coeficientes obtenidos para el caso "hacia el conjunto de ecoparques".
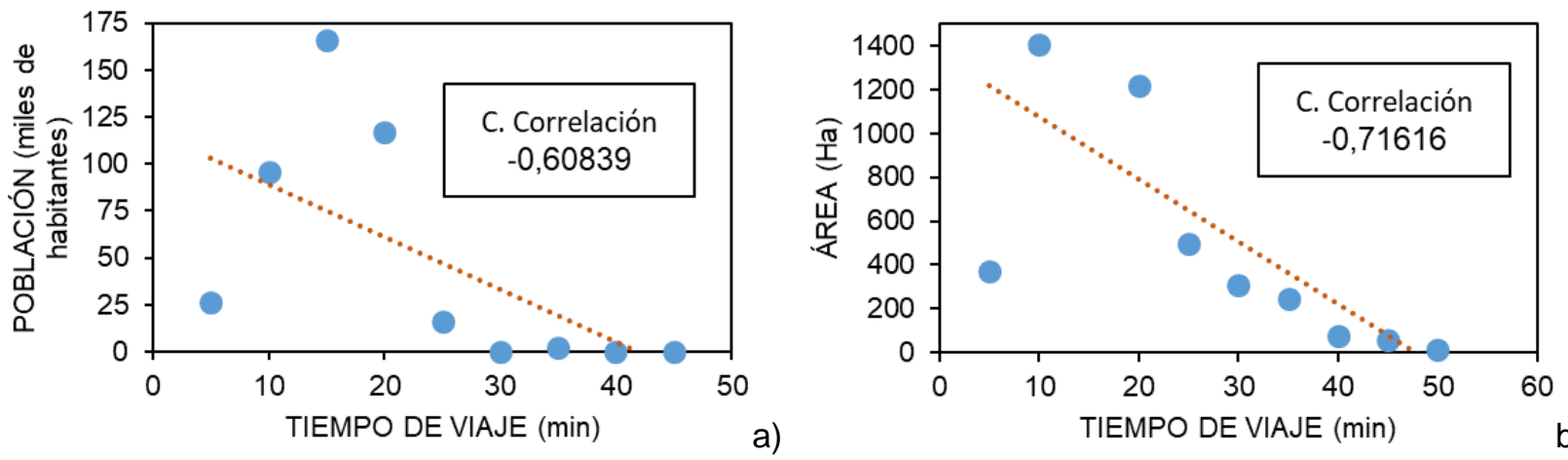

Fig. 12: Coeficientes de correlación, caso "hacia los ecoparques", a) población vs tiempo medio de viaje; b) área vs tiempo medio de viaje. 
El coeficiente de correlación entre las variables población y tiempo medio de viaje (Ver figura 12a), es de 0,60839 , que representa una relación decreciente entre ambas variables, mientras el tiempo medio de viaje aumente, la población cubierta disminuye; igual comportamiento se observa al correlacionar las variables área y tiempo medio de viaje (Ver figura 12b), con un coeficiente de correlación de -0,71616. Al igual que en el caso "hacia los ecoparques", se identifican correlaciones decrecientes, pero con valores más bajos; no obstante, se sigue encontrando que existe una relación entre tiempo medio de viaje y cobertura de las variables población y área dada la localización geoespacial de los ecoparques, existente y significativa.

\section{Análisis de accesibilidad territorial por cuadrantes}

Como complemento del análisis de accesibilidad territorial por estratos socioeconómicos, se estructura el análisis a partir de los centros de gravedad poblacional de cada comuna en la zona de estudio; esta evaluación permite establecer la condición de acceso por sectores en la zona de estudio mediante el uso del tiempo medio de viaje promedio de cada cuadrante. Como resultado se obtiene la figura 13, en la cual se aprecia la variación en tiempo de desplazamiento desde los ecoparques a cada cuadrante y comuna, se establece un máximo de 32,5 minutos sobre el sector noroccidental asociado al cuadrante 1 de la comuna 1; de igual forma, se aprecia un requerimiento en tiempo importante sobre el flanco sur-occidental, sobre el municipio de Villamaría en un tiempo de hasta 25 minutos.

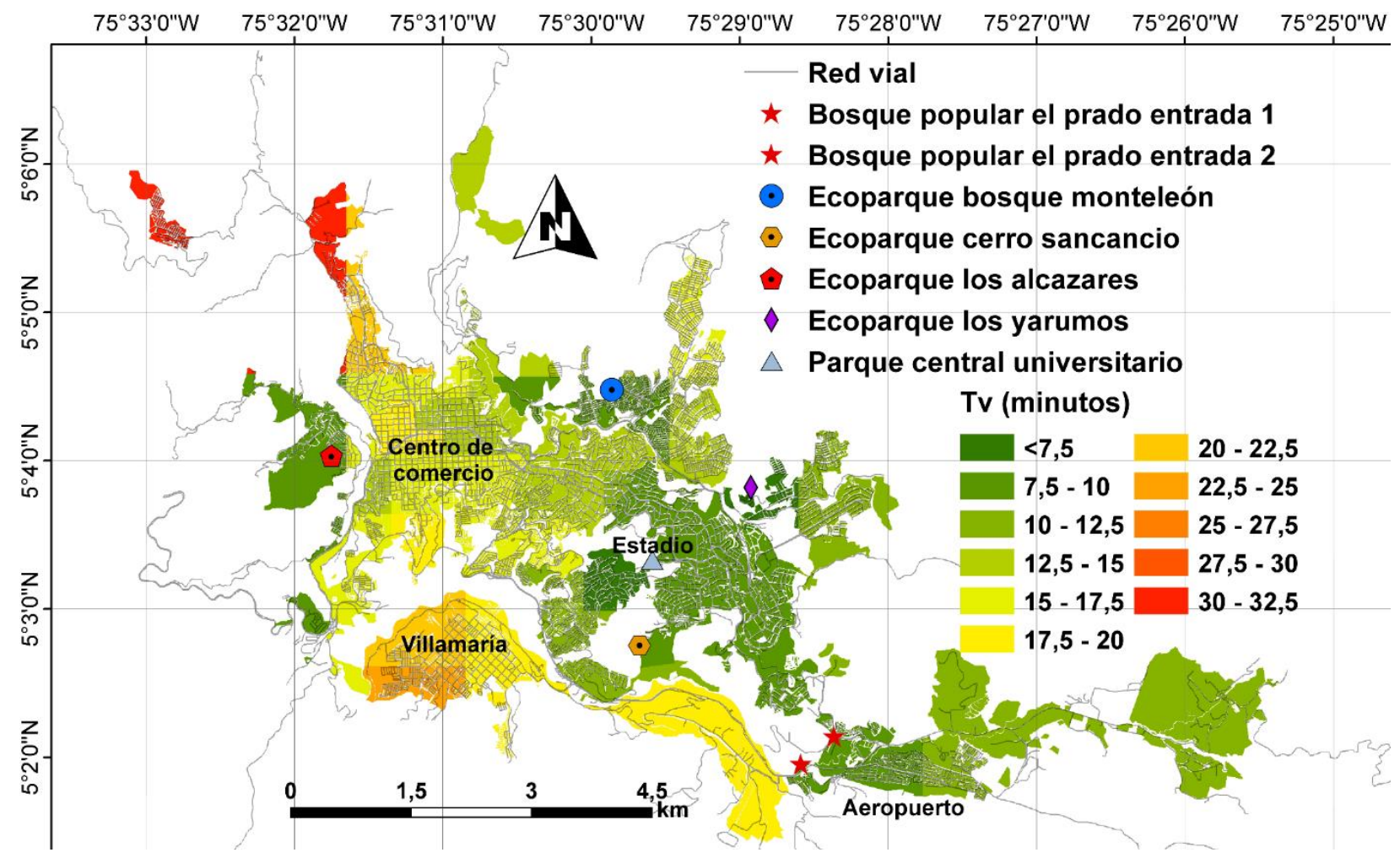

Fig. 13: Análisis de accesibilidad territorial desde los ecoparques a los centros de gravedad poblacional.

Por su parte, la figura 14 se observa un comportamiento similar al de la figura 13, resaltándose una variación notoria en el sector centro de comercio de la ciudad; no obstante, a pesar de esta pequeña variación, es posible afirmar la necesidad de incluir o fomentar la construcción de centros ecoturísticos sobre estas zonas, esto con el fin de incluir o disminuir el tiempo de acceso de los usuarios residentes en el sector, así como la recuperación y resguardo de zonas con complicaciones ambientales.

\section{Análisis individual de cobertura}

Con el fin de caracterizar el comportamiento en la cobertura ofrecida por cada uno de los 6 ecoparques de la ciudad, se ejecuta un análisis individual mediante ojivas porcentuales de la cobertura ofrecida desde y hacia cada uno de éstos.

\section{Ecoparque Bosque Popular el Prado}

En la figura 15, se presenta el comportamiento de la cobertura poblacional y de área desde (a) y hacia (b) el ecoparque, es posible identificar que, para ambas evaluaciones, cerca del $60 \%$ de la población puede 
movilizarse en un tiempo de viaje inferior a los 25 minutos, de igual forma, la cobertura asociada al área de estudio, cuenta con disponibilidad de desplazarse desde y hacia alguno de los accesos del ecoparque en un tiempo de viaje menor a 30 minutos. El comportamiento cruzado de las curvas, infiere que, la disposición de la población es menos intensa en el intervalo de tiempo menor a 10 minutos, posterior a este tiempo, se alcanza focos poblacionales más densos.

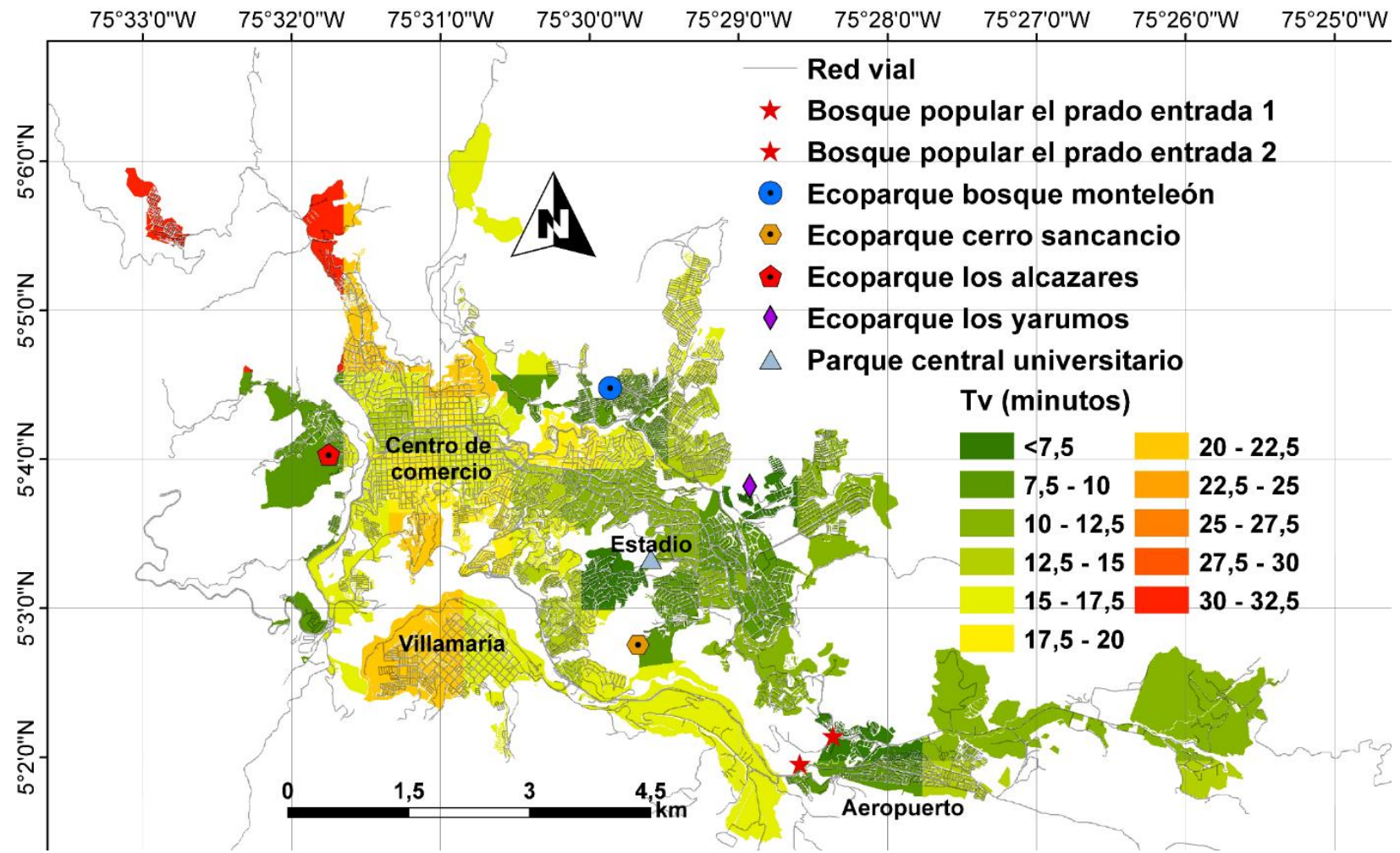

Fig. 14: Análisis de accesibilidad territorial hacia los ecoparques desde los centros de gravedad poblacional.
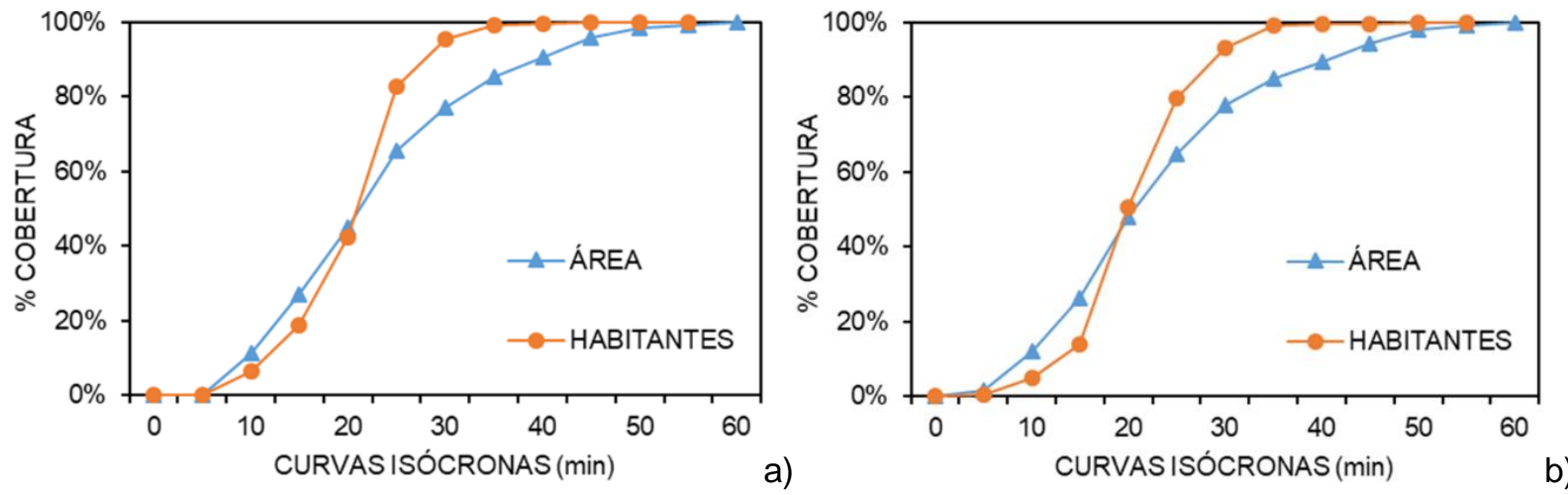

Fig. 15: Porcentaje acumulado de cobertura poblacional y de área en el ecoparque bosque popular el prado; a) desde el ecoparque; b) hacia el ecoparque.

Complementariamente, en la figura 16, se presenta el comportamiento en la cobertura por estrato socioeconómico, es posible observar que, la variación entre curvas desde (a) y hacia (b) el ecoparque, presenta una ligera variación, sin embargo, para ambos casos, el estrato con mayor facilidad de acceso es el número 6 , en el cual se requiere de un tiempo de viaje inferior al 15 minutos para cubrir el $50 \%$ de la población, seguidamente se encuentra el estrato número 5 con una pequeña alteración en su comportamiento desde y hacia el bosque popular, necesitando de cercano a los 17 minutos para suplir el mismo porcentaje de población. Por parte de los estratos con menor cobertura, se tiene que los números 1 y 3 requieren de un tiempo de viaje superior a los 20 minutos para suplir el $50 \%$ de su población, en ambos casos se presenta variación en el comportamiento de las curvas, formulando el estrato 1 como el de menor cobertura en los tiempos de viaje inferiores a los 20 minutos, y el estrato número 3 posterior a este valor. 

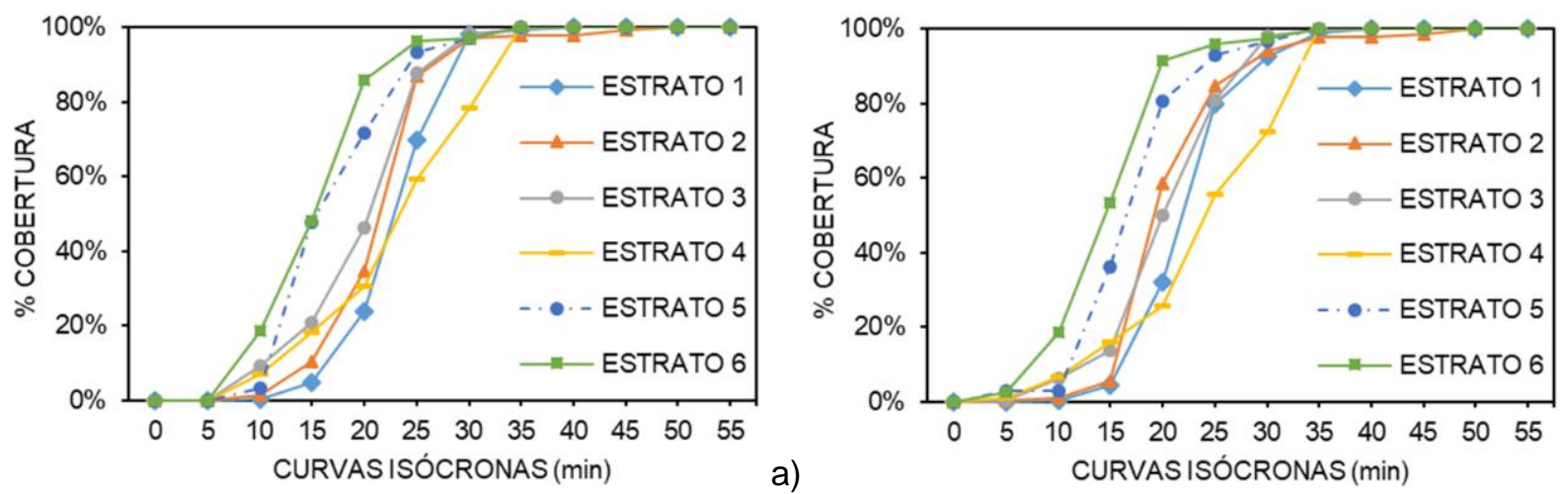

Fig. 16: Porcentaje acumulado de cobertura por estrato socioeconómico en el ecoparque bosque popular el prado a) desde el ecoparque; b) hacia el ecoparque.

\section{Ecoparque Los Yarumos}

La cobertura asociada al Ecoparque Los Yarumos (Figura 17), presenta un comportamiento inicial poco significativo, no obstante, la valoración del tiempo, requiere de un valor cercanos a los 25 y 30 minutos para suplir las necesidades del $50 \%$ de la población y área respectivamente, tanto en el escenario desde como hacia el ecoparque. De lado del análisis socioeconómico (Figura 18), se observa que, los estratos con mayor facilidad de acceso son los números 5 y 6 , los cuales requieren de un tiempo de viaje inferior a los 15 minutos para suplir cerca del $70 \%$ de ambas poblaciones; la variación registrada en relación a los estratos socioeconómicos es de alrededor de 10 minutos, siendo los estratos 1 y 3 los de mayor requerimiento para suplir el mismo porcentaje de cobertura.
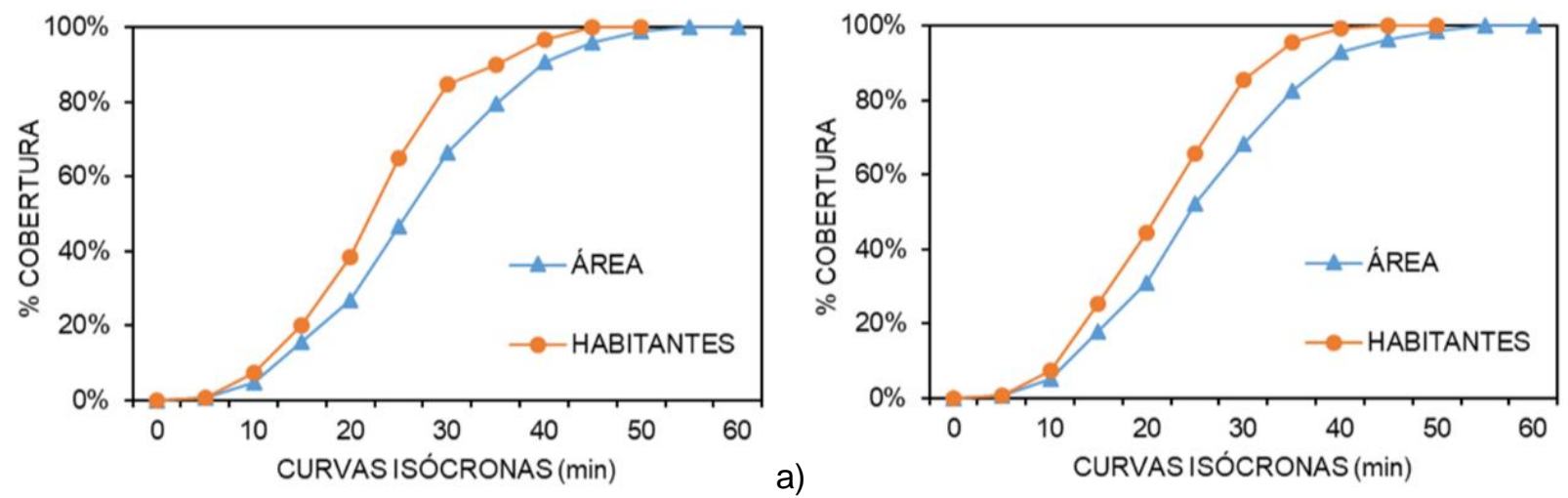

b)

Fig. 17: Porcentaje acumulado de cobertura poblacional y de área en el ecoparque los yarumos; a) desde el ecoparque; b) hacia el ecoparque.
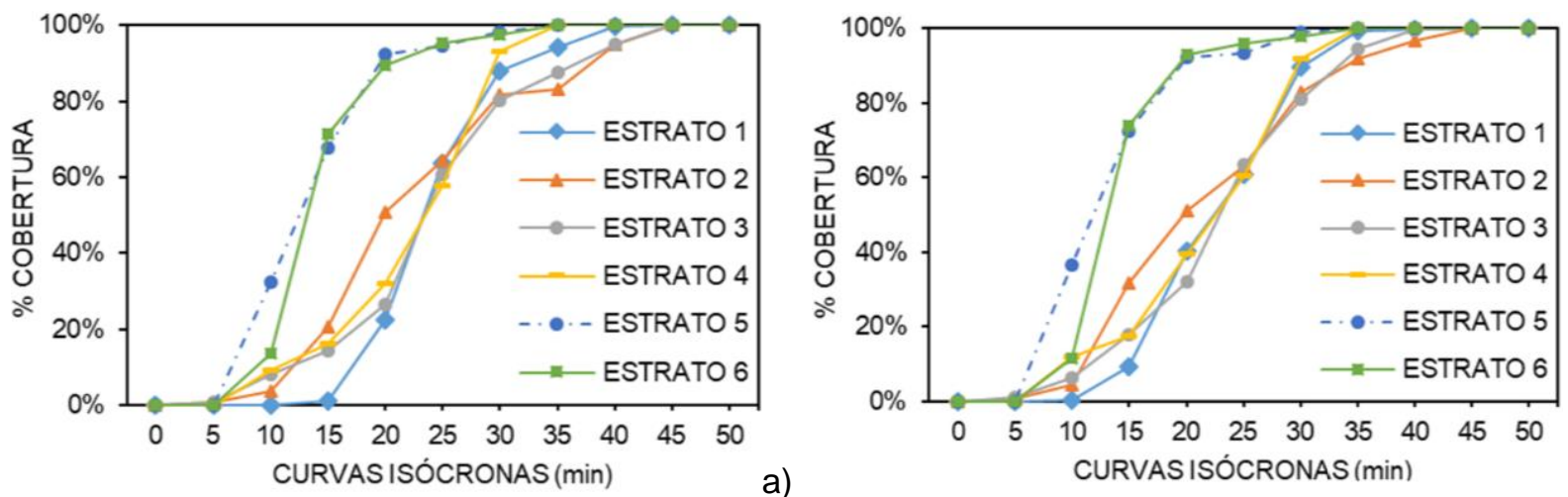

a)

b)

Fig. 18: Porcentaje acumulado de cobertura por estrato socioeconómico en el ecoparque los yarumos; a) desde el ecoparque; b) hacia el ecoparque.

Por otra parte, el comportamiento expresado por las curvas de cobertura, reafirman lo descrito en 2018 por Ios autores (Escobar et al., 2018b), dando pie a la posibilidad de recuperación y readecuación del ecoparque, 
motivo por el cual, el desarrollo del estudio, sirve de apoyo a la administración local como herramienta de planificación futura.

\section{Ecoparque Los Alcázares}

El comportamiento de área y población total asociado al Ecoparque Los Alcázares (Figura 19), presenta similitud de cobertura en ambas variables, para un tiempo inferior a los 20 minutos, este comportamiento refiere que, la población residente en cercanías al ecoparque se distribuye homogéneamente sobre la superficie aledaña; posterior a este valor, el comportamiento de las variables difiere en alrededor de 5 minutos, necesitando de alrededor de 30 minutos para cubrir el $50 \%$ de la población y 35 minutos para la cobertura de área.
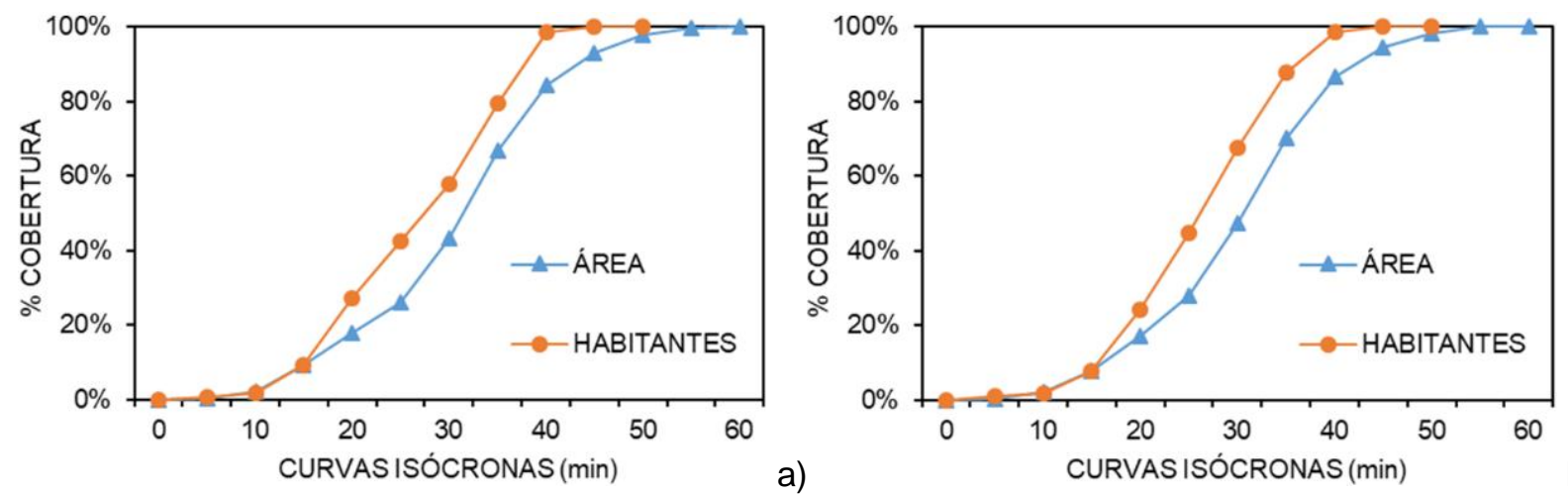

b)

Fig. 19: Porcentaje acumulado de cobertura poblacional y de área en el ecoparque los alcázares; a) desde el ecoparque; b) hacia el ecoparque.

En la figura 20, se presenta la variación en cobertura por estrato socioeconómico, como caso particular, se aprecia que, el estrato con mejor cobertura es el número 4, el cual requiere un tiempo de viaje cercano a los 22 minutos para suplir el $50 \%$ de la población en ambos escenarios. De otro lado, el comportamiento asociado al estrato con menor cobertura, se encuentra variación en los escenarios desde y hacia, en donde, para el primer caso, el estrato con mayor deficiencia es el número 2, con un tiempo de viaje cercano a 33 minutos para el $50 \%$ de cobertura; por parte del escenario hacía, se encuentra como el estrato menor servido, el número 6 , con un tiempo de viaje de alrededor de 32 minutos en el mismo valor cobertura.
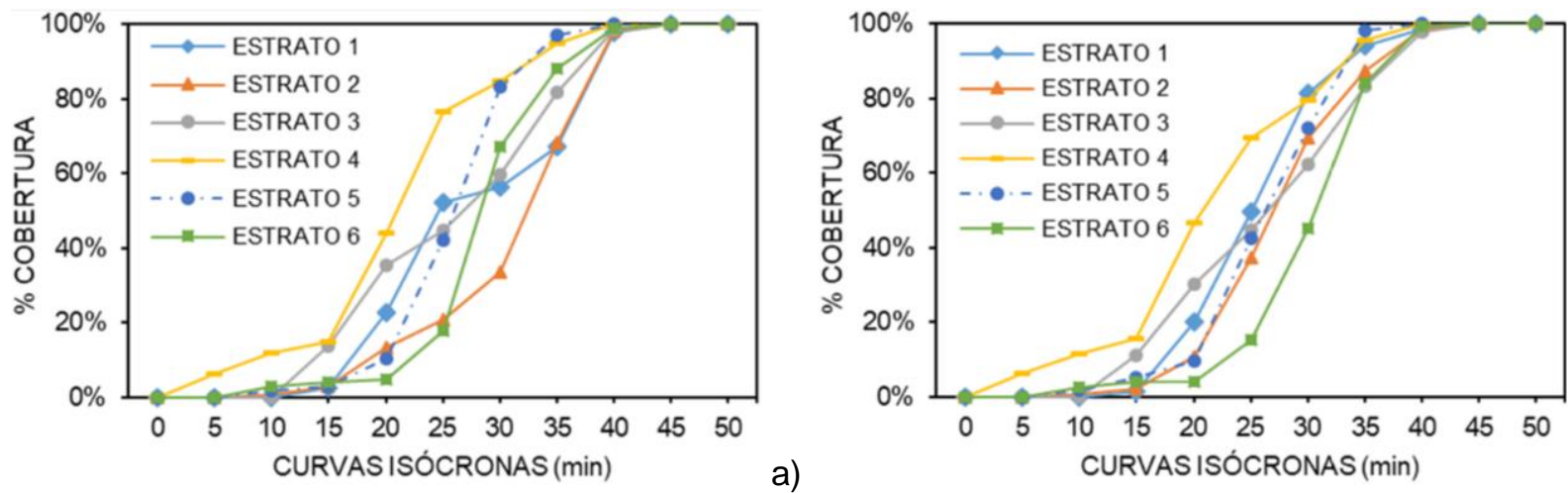

Fig. 20: Porcentaje acumulado de cobertura por estrato socioeconómico en el ecoparque los alcázares; a) desde el ecoparque; b) hacia el ecoparque.

\section{Ecoparque Central Universitario}

En la figura 21 se presenta el comportamiento en cobertura por población y área asociado al Ecoparque Central Universitario, el comportamiento entre curvas para ambos escenarios no presenta variación significativa, requiriendo de alrededor de 20 y 25 minutos para cubrir el $50 \%$ de la población y área respectivamente en ambos escenarios. La variación en cobertura por estrato socioeconómico (Figura 22) refleja un alto valor de la población cubierta para los estratos 5 y 6 , para los cuales, con un tiempo inferior a los 10 minutos se cubre hasta el $80 \%$ de la población. El comportamiento percibido, indica que los habitantes pertenecientes a estos estratos, residen en las cercanías, no obstante, sería necesario realizar un estudio más detallado para determinar a qué estrato pertenecen los visitantes de este ecoparque en particular. De lado de los estratos restantes, el comportamiento entre curvas es similar, no obstante, el estrato con menor 
cobertura es el número 1, el cual requiere de un tiempo de viaje cercano a los 20 minutos para alcanzar el $50 \%$ de su cobertura.
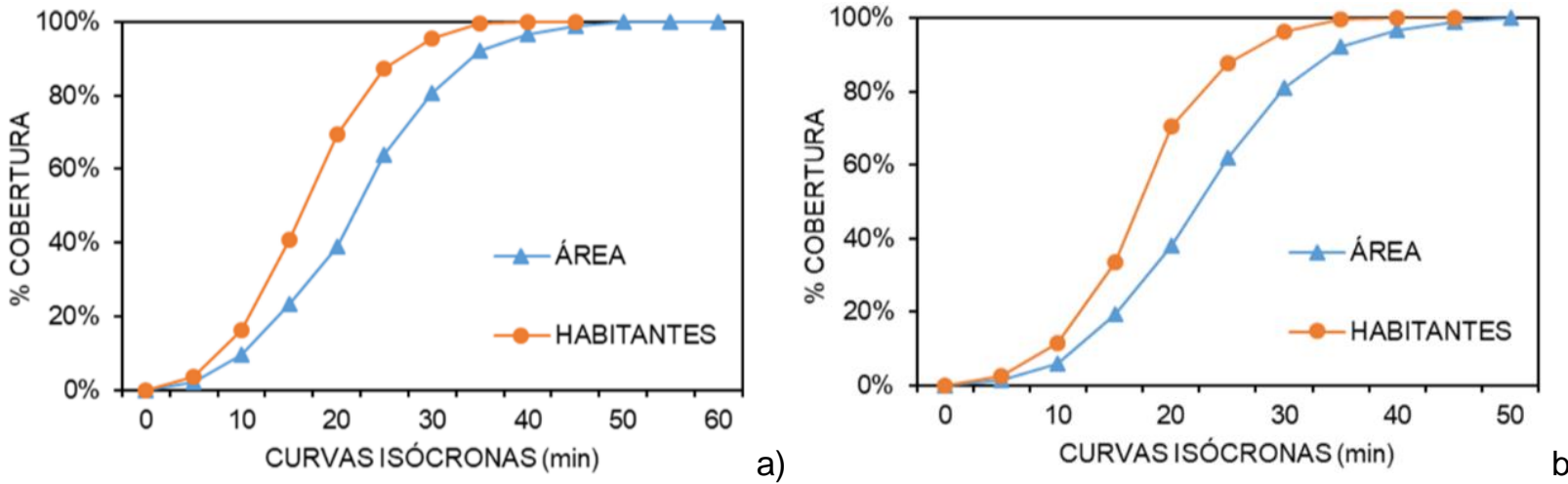

b)

Fig. 21: Porcentaje acumulado de cobertura poblacional y de área en el ecoparque central universitario; a) desde el ecoparque; b) hacia el ecoparque.
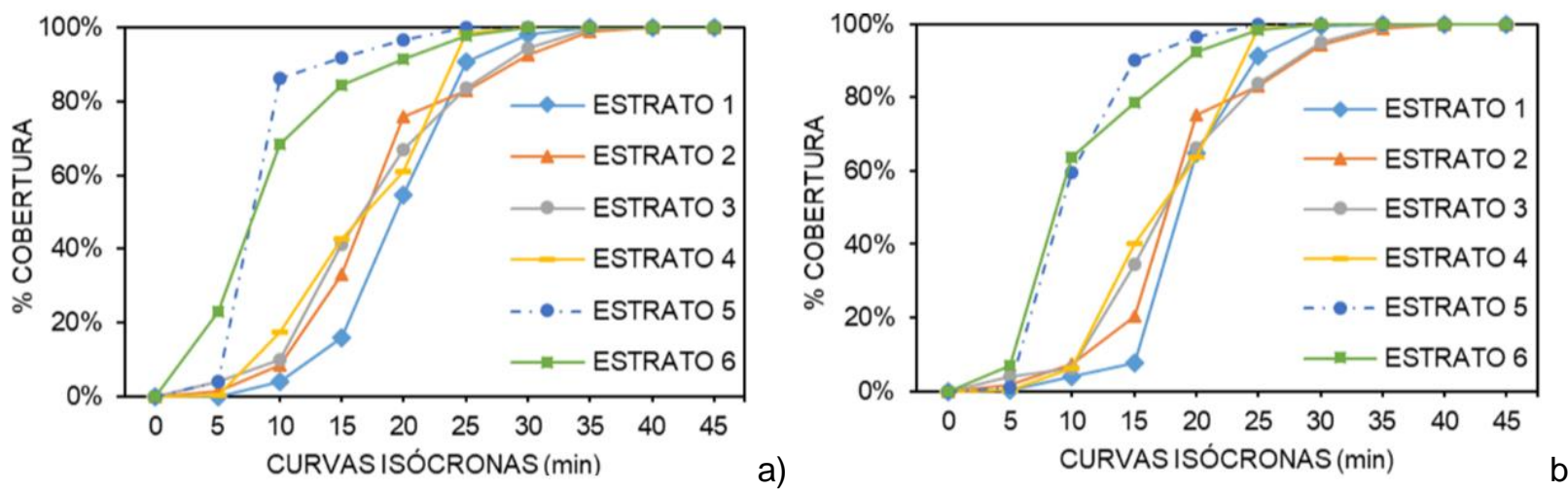

Fig. 22: Porcentaje acumulado de cobertura por estrato socioeconómico en el ecoparque central universitario; a) desde el ecoparque; b) hacia el ecoparque.

\section{Ecoparque Cerro Sancancio}

Como resultado de la evaluación del nivel de cobertura para el Ecoparque Cerro Sancancio, se obtiene la figura 23, en esta se aprecia que, para ambos escenarios, se requiere de un tiempo superior a los 20 minutos para lograr cubrir algún porcentaje significativo en población y área.
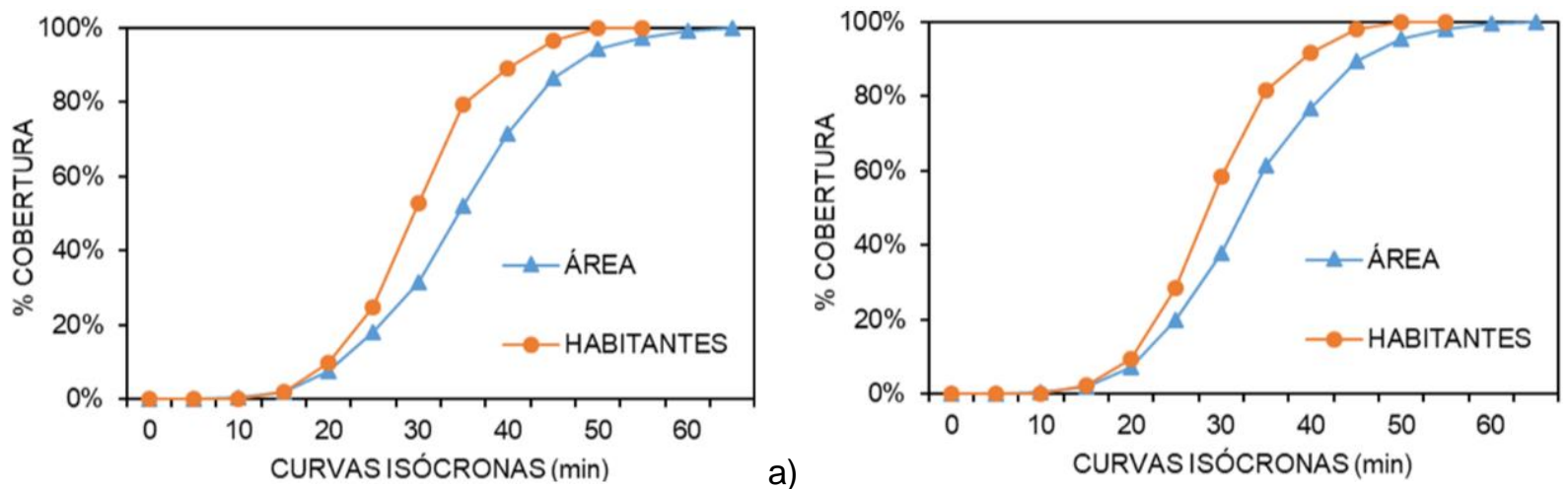

Fig. 23: Porcentaje acumulado de cobertura poblacional y de área en el ecoparque cerro sancancio; a) desde el ecoparque; b) hacia el ecoparque.

Por otra parte, el tiempo de viaje requerido para suplir el 50\% de la población y área es de 30 y 35 minutos respectivamente para ambos escenarios. En la figura 24, se presenta el comportamiento asociado a la cobertura poblacional por estrato socioeconómico del Ecoparque Cerro Sancancio, se tiene como población de mayor cobertura, los estratos 5 y 6 , los cuales requieren de un tiempo de viaje inferior a 20 minutos para suplir el $50 \%$ de la población, por su parte, los estratos 1, 2, 3 y 4, se comportan de forma similar, necesitando de alrededor de 30 minutos para cubrir el mismo porcentaje de cobertura. 

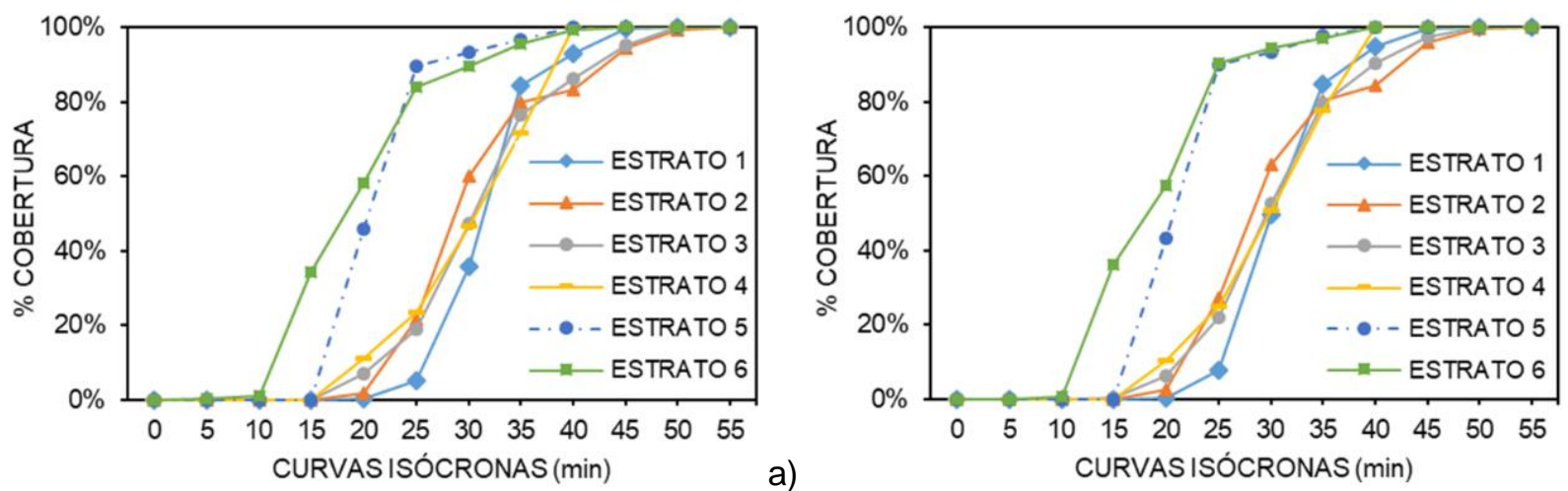

Fig. 24: Porcentaje acumulado de cobertura por estrato socioeconómico en el ecoparque cerro sancancio; a) desde el ecoparque; b) hacia el ecoparque.

\section{Ecoparque Bosque Moteleón}

Finalmente, como resultado de la evaluación de cobertura del Ecoparque Bosque Monteleón, se construyen las figuras 25 y 26 , en las cuales se presentan los valores de cobertura asociados a la población y área servidas por el ecoparque. En la figura 25, se observa la valoración de área y población total, en donde se requiere de un tiempo de viaje superior a los 10 minutos para lograr cubrir un porcentaje significativo en cada variable; luego de este valor, el comportamiento entre curvas para cada escenario varia sutilmente, por otra parte, la separación de cobertura entre población y área se encuentra en un máximo de 8 minutos, con lo cual se obtiene que, para el $50 \%$ de cobertura en el escenario desde el ecoparque, se necesita de un tiempo de viaje de 20 y 28 minutos para suplir la población y área. De lado del escenario hacia el ecoparque, la variación entre curvas es menor (5 minutos), sin embargo, el requerimiento en tiempo de viaje para suplir el mismo porcentaje de cobertura, es de 30 y 35 minutos respectivamente.
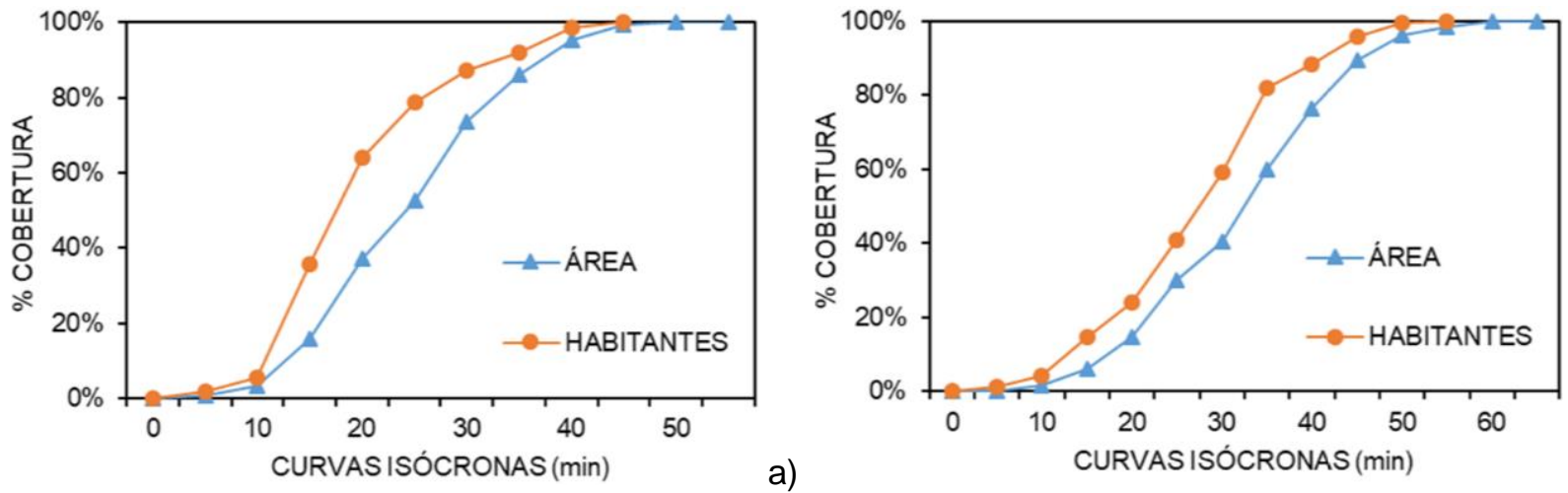

b)

Fig. 25: Porcentaje acumulado de cobertura poblacional y de área en el ecoparque bosque monteleón; a) desde el ecoparque; b) hacia el ecoparque.

De lado de la figura 26, se observa la variación en cobertura generada por estrato socioeconómico, es posible observar una fuerte variación entre los escenarios desde y hacia, para el caso inicial, se parecía poca separación entre curvas, con lo cual en un tiempo de viaje menor a los 17 minutos es posible suplir el $50 \%$ de la población en cada estrato; de lado del escenario hacia el ecoparque, se aprecia una separación mayor entre curvas, marcando como estrato de mayor cobertura en la fase inicial, el estrato número 2, sin embargo, posterior a los 17 minutos, la población de los estratos 5 y 6 , logran acceder fácilmente al ecoparque.

A pesar de la amplia información obtenida como resultados, se encontraron algunas falencias durante el proceso investigativo, falencias que, si bien no implican riesgo en el desarrollo metodológico, si consumen gran cantidad de tiempo y formulaciones para ser sorteadas. Algunas de las dificultades percibidas durante el proceso, se basan en la estructuración de la red de infraestructura, la cual, a pesar de contar con un alto detalle, presentaba falencias en cuanto a conectividad, asociadas a la vinculación de nuevos proyectos de infraestructura, además de posibles desplazamientos de la red en los procesos de edición; por otra parte, el proceso de obtención y formulación de cuadrantes, presenta fuertes complicaciones asociadas directamente a la obtención de información, debido a la baja disponibilidad de la cartografía asociada a la distribución espacial de la población, no obstante, luego de un amplio proceso de búsqueda, se logra obtener información base de la administración municipal, la cual es complementada con información de entidades gubernamentales externas. 

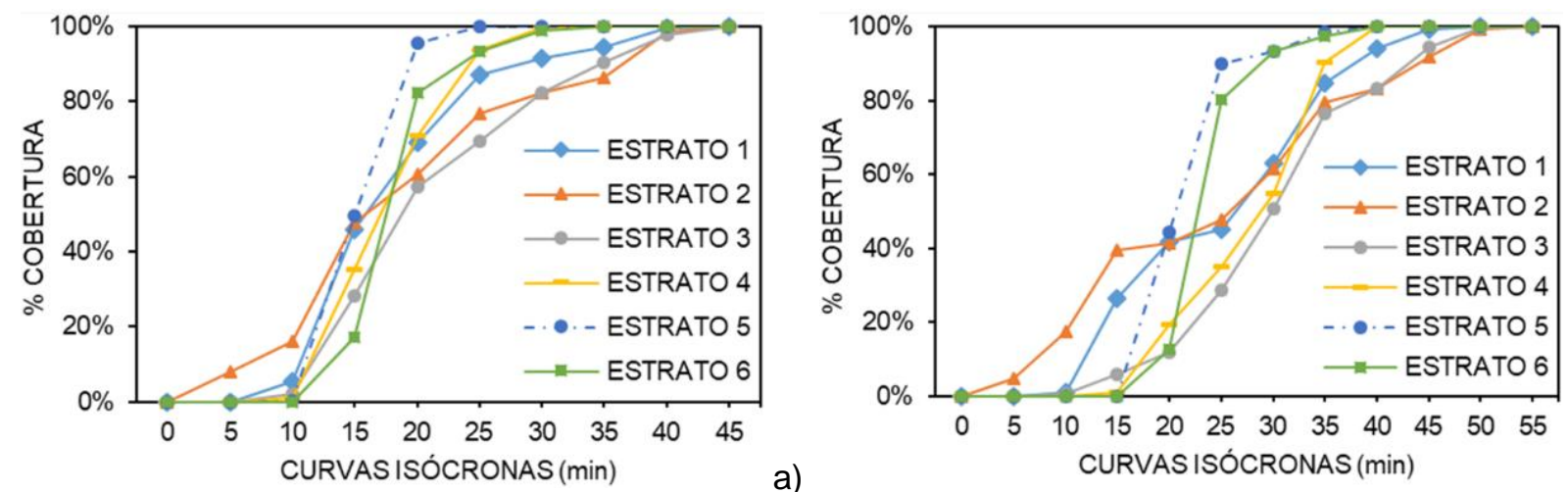

Fig. 26: Porcentaje acumulado de cobertura por estrato socioeconómico en el ecoparque bosque monteleón; a) desde el ecoparque; b) hacia el ecoparque.

De igual forma, es posible emplear como complemento, análisis de comportamientos de los usuarios de los centros ecoturísticos, permitiendo evaluar los patrones de movimientos (Lau y McKerher, 2006; Paulino et al., 2019), con los cuales se podría estudiar con mayor detalle la influencia de cada uno de los ecoparques sobre la comunidad y sobre el territorio como tal.

\section{CONCLUSIONES}

Las condiciones de accesibilidad territorial que tendría la población puede considerarse bueno, teniendo en cuenta que el tiempo medio de desplazamiento que se invierte en otras ciudades de Colombia superan los 55 minutos, mientras que en nuestro caso de estudio los valores obtenidos son menores; aclarando que, para nuestro modelo de análisis, se consideraron el promedio de las velocidades de todos los modos de transporte que circulan sobre la red de infraestructura de transporte de la zona de estudio. El análisis individual re afirma la condición de beneficio para los estratos 5 y 6, a excepción del Ecoparque los Alcázares, el cual posibilita con mayor facilidad el acceso a los habitantes de estrato 4; sin embargo, en cada uno de los centros ecoturísticos, se ve marginada la población de estratos inferiores, por lo cual se debe buscar una medida de mayor inclusión para los usuarios de menor condición socioeconómica; no obstante, se tienen una amplia cobertura poblacional en cada modo de evaluación, en donde más del $70 \%$ de la población accede o evacúa los ecoparques en un tiempo de desplazamiento inferior a los 15 minutos.

Así mismo, a pesar de observarse una amplia cobertura poblacional respecto al conjunto de ecoparques, se evidencia una alta concentración sobre el flanco occidental de la ciudad, motivo por el cual se podría emplear la metodología como mecanismo de orientación de la implementación de nuevos entornos ecoturísticos, no obstante, dadas las problemáticas de expansión asociadas al desarrollo de una ciudad, se podría buscar la forma de implementación de políticas públicas que garanticen la preservación de espacios verdes en los sectores de mayor deficiencia.

Por otra parte, el análisis por cuadrante sectoriza de una manera más detallada las zonas con mayores falencias asociadas al desplazamiento en el entorno urbano ya estructurado, esta valoración detallada, facilita los procesos de intervención y priorización en materias de movilidad, permitiendo a la administración local observar los sectores con requerimientos prioritarios y focalización de recursos.

Adicionalmente, es posible contemplar la interacción de la metodología de investigación en otros procesos de análisis, en los cuales se fomente el uso de los ecoparques como medidas de mitigación del estrés o condiciones particulares de salud, así como el grado de contaminación percibido en los entornos aledaños a las áreas protegidas, generando un mayor impacto social y medioambiental.

\section{REFERENCIAS}

Batty, M., Accessibility: in search of a unified theory, https://doi:10.1068/b3602ed, Environment and Planning B, Planning and Design, 36(2), 191-194 (2009).

Bertolini, L., Clercq, F. y Kapoen, L., Sustainable accessibility: a conceptual framework to integrate transport and land use plan-making. Two test-applications in the Netherlands and a reflection on the way forward, https://doi:10.1016/j.tranpol.2005.01.006, Transport Policy, 12(3), 207-220 (2005).

Bogotá Como Vamos - BCV, La movilidad en Bogotá, un compromiso, (2017).

Boisjoly, G. y Ahmed, M., The insider: A planners perspective on accessibility, https://doi.org/10.1016/j.jtrangeo.2017.08.006, Journal of Transport Geography, 64, 33-43 (2017). 
Cao, M., Shao, L. y Peng, L., Analysis of the network of protected areas in china based on a geographic perspective: current status, issues and integration, https://doi:10.3390/su71115617, Sustainability 7, 15617-15631 (2015).

Dijstra, E.W., A note on two problems in connexion with graphs, https://doi.org/10.1007/BF01386390, Numerical Mathematics, 1(1), 269-271 (1959).

Escobar, D.A., Montoya, J.A. y Galindo, J.A., Plan of intervention in the transport infrastructure network as a measure of heritage conservation. case study: la camelia ropeway station and tower of Herveo-Manizales, Colombia, https://doi.org/10.12988/ces.2018.87302, Contemporary Engineering Sciences, 11(58), 2887-2902 (2018).

Escobar, D.A., Montoya, J.A. y Moncada, C.A., Evolution of urban accesibility in Manizales, Colombia, 2010 - 2017, doi:10.17485/ijst/2018/v11i18/122745, Indian Journal of Science and technology, 11(18), (2018a).

Escobar, D.A., Montoya, J.A. y Moncada, C.A., The Yarumos Eco-Park: Evaluation of its Current Situation with Emphasis on Territorial Accessibility, https://doi:10.5539/mas.v12n7p89, Modern Applied Science, 12(7), (2018b).

Franco, Á. y S. Zabala, Los Equipamientos Urbanos Como Instrumentos para la Construcción de Ciudad y Ciudadanía, Uniandes, Dearq, Bogotá, 11, 8-13 (2012).

Geurs, K.T. y Van Wee, B., Accessibility evaluation of land-use and transport strategies: review and research directions, https://doi:10.1016/j.jtrangeo.2003.10.005, Journal of Transport Geography, 12(2), 127-140 (2004).

Gross, J.L. y Tucker, T.W., Topological Graph Theory, Dover Publications, (1987).

Guzmán, L.A., Escobar, F., Peña, J. y Cardona, R., A cellular automata-based land-use model as an integrated spatial decision support system for urban planning in developing cities: The case of the Bogotá region, https://doi.org/10.1016/j.landusepol.2019.104445, Land Use Policy, 92, 1-13 (2020).

Hansen, W.G., How accessibility shapes land use, https://doi:10.1080/01944365908978307, Journal of the American Institute of Planners, 25(2), 73-76 (1959).

Lau, G. y McKercher, B., Understanding tourist movement patterns in a destination: $A$ GIS approach, https://doi:10.1057/palgrave.thr.6050027, Tourism and Hospitality Research, 7(1), 39-49 (2006).

Londoño, A., Escobar, D.A. y Moncada, C.A., Metodología cualitativa y cuantitativa para calificación y priorización en auditorías de seguridad vial, Revista Espacios, ISSN 0798-1015, 30(52), 6 (2017).

Moncada, C.A., Cardona, S. y Escobar, D.A., Saving travel time as an urban planning instrument. case study: Manizales, Colombia, https://doi:10.5539/mas.v12n6p44, Modern Applied Science, 12(6), 44-57 (2018).

Morris, J.M., Dumble, P.L. y Wigan, M.R., Accessibility indicators for transport planning, https://doi:10.1016/01912607(79)90012-8, Transportation Research Part A: General, 13(2), 91-109 (1979).

MOOVIT, Global cities public transit usage report, (2016).

Mozón de Cáceres, A., La Accesibilidad individual como elemento de evaluación de los planes de transporte en la comunidad de Madrid/España, Informes de la Construcción, ISSN 0020-0883, 40(396), 21-38 (1988).

Newland, C.P., Zecchin, A.C., y otros tres autores, Empirically derived method and software for semi-automatic calibration of Cellular Automata land-use models, https://doi:10.1016/j.envsoft.2018.07.013, Environmental Modelling \& Software, 108, 208-239 (2018).

Oldfield, T.E., Smith, R.J., Harrop, S.R. y Leader, N., A gap analysis of terrestrial protected areas in England and its implications for conservation policy, https://doi:10.1016/j.biocon.2004.03.003, Biological Conservation, 120(3), 303-309 (2004).

Paquot, T., Releer el derecho a la ciudad de Henri Leferbvre, Articulos y Notas de Investigación, Urban, NS02, 81-87 (2012).

Paulino, I., Prats, L. y Whalley, P.A., Establishing Influence Areas of Attractions in Rural Destinations. http://doi:10.1080/21568316.2019.1673811, Tourism Planning \& Development, 1-25 (2019).

Sallán, J., Guardiet, J. y Suñé, A., Métodos cuantitativos de organización industrial I, Barcelona, Ediciones de la Universidad Politécnica de Catalunya, 159 (2010).

Schürmann, C., Spiekermann, K. y Wegener, M., Accessibility indicator, SASI Deliverable D5, Report to the European Commission, Berichte aus dem Institut für Raumplanung, Dortmund, 39, (1997).

Unal, M., Uslu, C. y Cilek, A., GIS-Based Accessibility for Neighbourhood Parks: The Case of Cukurova District, https://doi:10.14627/537612006, Journal of Digital Landscape Architecture, 1, 46-56 (2016).

Van Lindert, P., Rethinking urban development in Latin America: a review of changing paradigms and policies, https://doi:10.1016/j.habitatint.2015.11.017, Habitat International, 54(3), 253-264 (2016)

Vega, A., A multi-modal approach to sustainable accessibility in Galway, https://doi:10.1080/20429843.2011.9727923, Regional Insights, 2(2), 15-17 (2011).

Wu J, Feng, Z. y otros tres autores, Neglected Green Street Landscapes: A Re-Evaluation Method of Green Justice, https://doi:10.1016/j.ufug.2019.05.004, Urban Forestry and Urban Greening, 41, 344-353 (2019). 